\title{
Secure Massive MIMO Transmission with an Active Eavesdropper
}

\author{
Yongpeng Wu, Member, IEEE, Robert Schober, Fellow, IEEE, Derrick Wing Kwan Ng, Member, IEEE, \\ Chengshan Xiao, Fellow, IEEE, and Giuseppe Caire, Fellow, IEEE.
}

\begin{abstract}
In this paper, we investigate secure and reliable transmission strategies for multi-cell multi-user massive multipleinput multiple-output (MIMO) systems with a multi-antenna active eavesdropper. We consider a time-division duplex system where uplink training is required and an active eavesdropper can attack the training phase to cause pilot contamination at the transmitter. This forces the precoder used in the subsequent downlink transmission phase to implicitly beamform towards the eavesdropper, thus increasing its received signal power. Assuming matched filter precoding and artificial noise (AN) generation at the transmitter, we derive an asymptotic achievable secrecy rate when the number of transmit antennas approaches infinity. For the case of a single-antenna active eavesdropper, we obtain a closed-form expression for the optimal power allocation policy for the transmit signal and the $\mathrm{AN}$, and find the minimum transmit power required to ensure reliable secure communication. Furthermore, we show that the transmit antenna correlation diversity of the intended users and the eavesdropper can be exploited in order to improve the secrecy rate. In fact, under certain orthogonality conditions of the channel covariance matrices, the secrecy rate loss introduced by the eavesdropper can be completely mitigated.
\end{abstract}

\section{INTRODUCTION}

The emergence of smart mobile devices such as smart phones and wireless modems has led to an exponential increase in the demand for wireless data services. A recent and promising solution to meet this demand is massive multipleinput multiple-output (MIMO) technology, which utilizes a very large number of antennas and simple signal processing at the base station (BS) to serve a comparatively small (with respective to the number of antennas) number of users. The field of massive MIMO communication systems was initiated

Copyright (c) 2014 IEEE. Personal use of this material is permitted. However, permission to use this material for any other purposes must be obtained from the IEEE by sending a request to pubs-permissions@ieee.org

This paper was presented in part at IEEE ICC 2015.

The work of $\mathrm{Y}$. Wu and R. Schober was supported by the Alexander von Humboldt Foundation and the German Science Foundation (Grant SCHO831/5-1). The work of C. Xiao is supported in part by US National Science Foundation under grant ECCS-1231848. The work of G. Caire is supported by the Alexander von Humboldt Foundation.

Y. Wu and R. Schober are with Institute for Digital Communications, Universität Erlangen-Nürnberg, Cauerstrasse 7, D-91058 Erlangen, Germany (Email: yongpeng.wu@fau.de; robert.schober@fau.de;).

D. W. K. Ng is with the School of Electrical Engineering and Telecommunications, University of New South Wales, Sydney, N.S.W., Australia (E-mail: w.k.ng@unsw.edu.au).

C. Xiao is with the Department of Electrical and Computer Engineering, Missouri University of Science and Technology, Rolla, MO 65409, USA (Email: xiaoc@mst.edu).

G. Caire is with Institute for Telecommunication Systems, Technical University Berlin, Einsteinufer 25, 10587 Berlin, Germany (Email: caire@ @uberlin.de) by the pioneering work in [1] which considered multi-cell multi-user time-division duplex (TDD) communication. The key idea in [1] is that as the number of transmit antennas increases, the effects of uncorrelated receiver noise and fast fading vanish due to the law of large numbers. Then, the only residual interference is caused by the reuse of the same pilot sequences in adjacent cells. This effect is known as pilot contamination. Since the publication of [1], a considerable amount of research has been dedicated to studying various aspects of massive MIMO systems [2-10]. In particular, A. Adhikary et al. and C. Sun et al. design transmission schemes to serve different users in orthogonal spatial resources by exploiting the unique property of the massive MIMO channels in [4] and [8], respectively, which inspire a null space design for secure massive MIMO transmission in this paper. Also, massive MIMO has been investigated for various types of systems, including: Single-cell multi-user uplink/downlink systems [11-13], multi-cell multi-user uplink/dowlink systems $[14,15]$, orthogonal frequency-division multiple access systems [16-18], non-orthogonal multiple access systems [19], and systems employing constant-envelope signals [20].

The broadcast nature of the wireless channel makes it inherently prone to security breaches such as eavesdropping and jamming, which jeopardizes the privacy of communication in wireless networks. In order to maintain the required level of privacy, appropriate signal and information processing techniques have to be employed to ensure reliable and secure communication. Traditional approaches to secure communication for preventing unauthorized reception by eavesdroppers rely on cryptographic encryption implemented in the application layer. These methods may entail a relatively high complexity due to the required key distribution and service management [21]. As a complement to cryptographic methods, physical layer security, which considers communication security from an information-theoretic perspective, has attracted significant research interest recently. In Wyner's pioneering work on information-theoretic security, a "wiretap channel" model was defined along with the associated secrecy capacity [22]. Wyner's work indicates that the transmitter can reliably send a private message to the receiver, which cannot be decoded by the eavesdropper, if the channel of the eavesdropper is a degraded version of the channel of the desired receiver. Wyner's result was extended to more general non-degraded channels in [23]. More recent studies have investigated the capacity and precoder design for multi-antenna wiretap channels [2432]. In particular, if only imperfect channel state information (CSI) of the eavesdropper is available at the transmitter, it is 
advantageous to transmit artificial noise (AN) along with the information-carrying signal to interfere the decoding process at the eavesdropper [33-36]. In [37], the authors propose a thorough analysis and optimization framework for artificial noise assisted secure transmission in a MIMO wiretap channel.

Physical layer security for massive MIMO systems with passive eavesdroppers has been recently studied. The authors in [39] first applied the technique of large-scale antenna array into physical layer security, and investigate the limiting performance while the number of antennas approached infinity. Secure massive MIMO transmissions for multi-cell multiuser systems with imperfect CSI have been investigated in $[40,41]$, where a passive eavesdropper attempts to decode the information sent to one of the users. In [42], a comprehensive performance analysis of AN aided multi-antenna secure transmission in multi-cell multi-user systems under a stochastic geometry framework is provided. In [39-42], it was assumed that the channel gains of both the desired receiver and the eavesdropper are independent and identically distributed (i.i.d.).

Most existing studies on physical layer security assume that perfect CSI of the legitimate channel is available at the transmitter and do not consider the channel training phase required to acquire the CSI. However, in TDD communication systems, the BS needs to estimate the channel for the subsequent downlink transmission based on pilot sequences sent by the users in an uplink training phase. Furthermore, the low rank property of massive MIMO channels has been exploited in [38] to significantly save the pilot training overhead and reduce the signal processing complexity at users to achieve reliable CSI at the BS. As a result, a smart eavesdropper might actively attack this channel training phase by sending the same pilot sequences as the users to cause pilot contamination at the transmitter, which improves the eavesdropping capability of the eavesdropper significantly [43].

Hence, the so-called pilot contamination attack poses a serious secrecy threat to TDD-based massive MIMO systems. In such systems, the channel hardening due to beamforming with large antenna arrays [2] makes the exploitation of statistical fluctuations due to fading for secrecy enhancement impossible. Furthermore, the pilot contamination attack directs the transmitter beamforming to the advantage of the eavesdropper. Therefore, for a sufficiently large eavesdropper pilot power, the achievable secrecy rate may approach zero. This goes against the conventional wisdom [5] that massive MIMO inherently facilitates secure communication because the base station can form very narrow beams focusing on the target users and therefore avoiding spill over of the signal power in other directions.

A single cell massive MIMO system with an active eavesdropper was investigated for i.i.d. fading channels [44-46]. However, systematic approaches for combating the pilot contamination attack of a multi-antenna active eavesdropper and maintaining secrecy of communication in correlated fading channels were not provided in [44-46] and have not been studied in the literature, yet.

In this paper, we study secure transmission over correlated fading channels in TDD multi-cell multi-user massive MIMO systems in the presence of a multi-antenna active eavesdropper. We assume that in the uplink training phase, the active eavesdropper sends the same pilot sequence as the desired receiver to impair the channel estimation at the transmitter, i.e., to cause pilot contamination at the transmitter. Subsequently, the transmitter uses the estimated channel for calculation of the precoder for downlink transmission. This paper makes the following key contributions:

1) We introduce a pilot contamination precoder, which allows the eavesdropper to optimize its attack. The proposed pilot contamination precoder is provided in closed form and maximizes the total average estimation error variance of the desired user's channel.

2) We derive a closed-form expression for the asymptotic achievable secrecy rate for TDD multi-cell multiuser massive MIMO systems employing matched filter precoding and $\mathrm{AN}$ generation (we refer to this design as MF-AN design) at the transmitter to combat a multi-antenna active eavesdropper. Based on the derived asymptotic expression, which is valid if the number of transmit antennas tends to infinity, the optimal power allocation policy for the information signal and the AN can be found by a simple one-dimension numerical search. Then, we show that, in the presence of an active eavesdropper, the secrecy rate is not a monotonically increasing function of the signal-to-noise ratio (SNR). For the special case of a single-antenna eavesdropper, we obtain the optimal power allocation policy for the transmit signal and the $\mathrm{AN}$ in closed form. In addition, we obtain the minimum transmit signal power required to ensure secure transmission.

3) For the case of correlated fading channels, we reveal that the impact of the active eavesdropper vanishes when the signal space (i.e., the span of the eigenvectors of the channel correlation matrix that correspond to nonzero eigenvalues) of the users and the eavesdropper are mutually orthogonal. Inspired by this observation, we exploit the low rank property of the transmit correlation matrices of massive MIMO channels [3, 4, 6-8, 10,38] to design an efficient precoding scheme that transmits in the null space (NS) of the transmit correlation matrix of the eavesdropper (we refer to this precoding solution as NS design). Unlike the conventional NS design for the perfect CSI case [24], the proposed NS design can completely remove the impact of the pilot contamination attack by performing joint uplink and downlink processing. For the special case of a single-antenna eavesdropper, we derive a threshold that can be used to determine whether the MF-AN design or the NS design is preferable for given channel and eavesdropper parameters.

4) We propose a unified design which combines the MFAN design and the NS design. Numerical results indicate that the proposed unified design can effectively mitigate the pilot contamination attack of an active eavesdropper in massive MIMO systems.

The remainder of this paper is organized as follows. In 
Section II, we introduce the adopted multi-cell multi-user massive MIMO system model with a multi-antenna active eavesdropper. In Section III, we derive an expression for the asymptotic achievable secrecy rate for the MF-AN design when the number of transmit antennas tends to infinity. Based on this expression, we derive transmission strategies to combat the pilot contamination attack from the active eavesdropper. In Section IV, we provide several novel insights for the singleantenna eavesdropper case. Numerical results are presented in Section V, and the main results are summarized in Section VI.

Notation: Vectors are denoted by lower-case bold-face letters; matrices are denoted by upper-case bold-face letters. Superscripts $(\cdot)^{T},(\cdot)^{*}$, and $(\cdot)^{H}$ stand for the matrix transpose, conjugate, and conjugate-transpose operations, respectively. We use $\operatorname{tr}(\mathbf{A})$ and $\mathbf{A}^{-1}$ to denote the trace operation and the inverse of matrix $\mathbf{A}$, respectively. $\|\cdot\|$ and $|\cdot|$ denote the Euclidean norm of a matrix/vector and a scalar, respectively. $\operatorname{diag}\{\mathbf{b}\}$ denotes a diagonal matrix with the elements of vector b on its main diagonal. vec $(\mathbf{A})$ stacks all columns of matrix A into a vector. The $M \times M$ identity matrix is denoted by $\mathbf{I}_{M}$, and the all-zero $M \times N$ matrix and $N \times 1$ vector are denoted by 0. The field of complex numbers is denoted by $\mathbb{C}$ and $E[\cdot]$ denotes statistical expectation. We use $\mathbf{x} \sim \mathcal{C N}\left(\mathbf{0}, \mathbf{R}_{N}\right)$ to denote a circularly symmetric complex Gaussian vector $\mathbf{x} \in \mathbb{C}^{N \times 1}$ with zero mean and covariance matrix $\mathbf{R}_{N}$. Based on [49, Definition II.1], we use $\mathbf{X} \sim \mathcal{C N}\left(\mathbf{0}, \mathbf{R}_{N} \otimes \mathbf{R}_{M}\right)$ to denote a circularly symmetric complex Gaussian matrix $\mathbf{X} \in \mathbb{C}^{N \times M}$ with zero mean and covariance matrix $\mathbf{R}_{N} \otimes \mathbf{R}_{M}$. $\{\mathbf{A}\}_{i j}$ returns the element of matrix $\mathbf{A}$ in the $i$ th row and the $j$ th column. $\mathbf{1}_{N}$ denotes an $N \times 1$ vector with all elements equal to 1 . $\mathbf{e}_{r}$ denotes the unit-vector with a one as the $r$ th element and zeros for all other elements. $[x]^{+}$stands for $\max \{0, x\}, \otimes$ denotes the Kronecker product, and $A \stackrel{N \rightarrow \infty}{\rightarrow} B$ means that $A$ converges almost surely to $B$ as $N$ goes to infinity.

\section{SySTEM MODEL}

We consider a multi-cell multi-user system with $L+1$ cells, cf. Figure 1. Each cell contains a BS equipped with $N_{t}$ antennas and $K$ single-antenna users. Without loss of generality, we denote the reference cell by $l=0$. An active eavesdropper with $N_{e}$ antennas (equivalent to $N_{e}$ cooperative single-antenna eavesdroppers) is located in the reference cell. The eavesdropper seeks to recover the private message intended for a specific target user $m$.

\section{A. Uplink Training and Channel Estimation}

In the uplink training and channel estimation phase, the received signal $\mathbf{Y}_{0} \in \mathbb{C}^{N_{t} \times \tau}$ at the $\mathrm{BS}$ in the reference cell is given by [3]

$$
\begin{aligned}
\mathbf{Y}_{0}=\sum_{k=1}^{K} \sqrt{P_{0 k}} \mathbf{h}_{0 k}^{0} \boldsymbol{\omega}_{0 k}^{T}+\sum_{l=1}^{L} \sum_{k=1}^{K} \sqrt{P_{l k}} \mathbf{h}_{l k}^{0} \boldsymbol{\omega}_{l k}^{T} \\
+\sqrt{\frac{P_{E}}{N_{e}}} \mathbf{H}_{E}^{0} \mathbf{P}_{e} \mathbf{W}_{e}+\mathbf{N}
\end{aligned}
$$

Here, $P_{l k}$ and $\boldsymbol{\omega}_{l k} \in \mathbb{C}^{\tau \times 1}$ are the average transmit power and the pilot sequence of the $k$ th user in the $l$ th cell, where $\tau$ denotes the length of the pilot sequence. $\mathbf{h}_{l k}^{p}=$ $\left(\mathbf{R}_{l k}^{p}\right)^{1 / 2} \mathbf{g}_{l k}^{p} \in \mathbb{C}^{N_{t} \times 1}$ denotes the channel vector between the $k$ th user in the $l$ th cell and the BS in the $p$ th cell, where $\mathbf{g}_{l k}^{p} \sim \mathcal{C N}\left(\mathbf{0}, \mathbf{I}_{N_{t}}\right)$ and $\mathbf{R}_{l k}^{p} \in \mathbb{C}^{N_{t} \times N_{t}}$ is the correlation matrix of channel $\mathbf{h}_{l k}^{p}$. $P_{E}$ denotes the average transmit power of the eavesdropper when attacking the uplink training. $\mathbf{H}_{E}^{l}=\left(\mathbf{R}_{E, T}^{l}\right)^{1 / 2} \mathbf{G}_{E}^{l}\left(\mathbf{R}_{E, R}^{l}\right)^{1 / 2} \in \mathbb{C}^{N_{t} \times N_{e}}$ denotes the channel between the eavesdropper and the BS in the $l$ th cell, where $\mathbf{G}_{E}^{l} \sim \mathcal{C N}\left(\mathbf{0}, \mathbf{I}_{N_{t}} \otimes \mathbf{I}_{N_{e}}\right)$. $\mathbf{R}_{E, T}^{l} \in \mathbb{C}^{N_{t} \times N_{t}}$ and $\mathbf{R}_{E, R}^{l} \in \mathbb{C}^{N_{e} \times N_{e}}$ are the transmit and receive correlation matrices of channel $\mathbf{H}_{E}^{l}$. The active eavesdropper attacks the channel estimation process of the $m$ th user in the reference cell by sending pilot contamination sequences $\mathbf{P}_{e} \mathbf{W}_{e}$, where $\mathbf{P}_{e} \in \mathbb{C}^{N_{e} \times N_{e}}$ is the pilot contamination precoder and $\mathbf{W}_{e}=\left[\boldsymbol{\omega}_{0 m}, \boldsymbol{\omega}_{0 m}, \cdots, \boldsymbol{\omega}_{0 m}\right]^{T} \in \mathbb{C}^{N_{e} \times \tau} . \mathbf{N} \in \mathbb{C}^{N_{t} \times \tau}$ is a Gaussian noise matrix with i.i.d. elements of zero-mean and variance $N_{0}$. The structure of the uplink received signal at the $\mathrm{BS}$ in the local cell is illustrated in Figure 2.

Eq. (1) can be rewritten as

$$
\begin{aligned}
\mathbf{y}_{0}=\sum_{k=1}^{K} \sqrt{P_{0 k}} & \left(\boldsymbol{\omega}_{0 k} \otimes \mathbf{I}_{N_{t}}\right) \mathbf{h}_{0 k}^{0} \\
& +\sum_{l=1}^{L} \sum_{k=1}^{K} \sqrt{P_{l k}}\left(\boldsymbol{\omega}_{l k} \otimes \mathbf{I}_{N_{t}}\right) \mathbf{h}_{l k}^{0} \\
& +\sqrt{\frac{P_{E}}{N_{e}}}\left(\boldsymbol{\omega}_{0 m} \otimes \mathbf{I}_{N_{t}}\right) \sum_{r=1}^{N_{e}} \mathbf{h}_{\mathrm{eff}, r}^{0}+\mathbf{n}
\end{aligned}
$$

where $\mathbf{y}_{0}=\operatorname{vec}\left(\mathbf{Y}_{0}\right), \mathbf{n}=\operatorname{vec}(\mathbf{N})$, and $\mathbf{h}_{\text {eff, } r}^{l}$ denotes the $r$ th column of matrix $\mathbf{H}_{E}^{l} \mathbf{P}_{e}, l=0,1, \cdots, L$. We assume that the same $K$ orthogonal pilot sequences are used by the $K$ users in each cell [1], i.e., $\boldsymbol{\omega}_{0 k}=\boldsymbol{\omega}_{1 k}=\cdots=\boldsymbol{\omega}_{L k}=\boldsymbol{\omega}_{k}$, $\boldsymbol{\omega}_{l k}^{H} \boldsymbol{\omega}_{l k}=\tau, \boldsymbol{\omega}_{l k}^{H} \boldsymbol{\omega}_{l p}=0, \forall k \neq p$. Then, the minimum mean square error (MMSE) estimate of $\mathbf{h}_{0 m}^{0}$ is given by [50]

$$
\begin{aligned}
\widehat{\mathbf{h}}_{0 m}^{0}=\sqrt{P_{0 m}} & \mathbf{R}_{0 m}^{0}\left(N_{0} \mathbf{I}_{N_{t}}+\tau\left(\sum_{t=0}^{L} P_{t m} \mathbf{R}_{t m}^{0}\right.\right. \\
& \left.\left.+P_{E} r_{E, R}^{0} \mathbf{R}_{E, T}^{0}\right)\right)^{-1} \widetilde{\mathbf{y}}_{0 m}
\end{aligned}
$$

where

$$
\begin{aligned}
\widetilde{\mathbf{y}}_{0 m}=\sqrt{P_{0 m}} & =\mathbf{h}_{0 m}^{0}+\sum_{t=1}^{L} \sqrt{P_{t m}} \tau \mathbf{h}_{t m}^{0} \\
& +\sqrt{\frac{P_{E}}{N_{e}}} \tau \sum_{r=1}^{N_{e}} \mathbf{h}_{\mathrm{eff}, r}^{0}+\left(\boldsymbol{\omega}_{m} \otimes \mathbf{I}_{N_{t}}\right)^{H} \mathbf{n}
\end{aligned}
$$

and $r_{E, R}^{l}=\frac{1}{N_{e}} \sum_{r=1}^{N_{e}} \sum_{s=1}^{N_{e}}\left\{\mathbf{P}_{e}^{H} \mathbf{R}_{E, R}^{l} \mathbf{P}_{e}\right\}_{r s}, \quad l=$ $0,1, \cdots, L$. The actual channel vector $\mathbf{h}_{0 m}^{0}$ can be written as $\mathbf{h}_{0 m}^{0}=\widehat{\mathbf{h}}_{0 m}^{0}+\mathbf{e}_{0 m}^{0}$, where the estimated channel $\widehat{\mathbf{h}}_{0 m}^{0} \sim \mathcal{C N}\left(\mathbf{0}, \widehat{\mathbf{R}}_{0 m}^{0}\right)$ and the estimation error $\mathbf{e}_{0 m}^{0} \sim$ $\mathcal{C N}\left(\mathbf{0}, \mathbf{R}_{0 m}^{0}-\widehat{\mathbf{R}}_{0 m}^{0}\right)$ are mutually independent. Correlation 


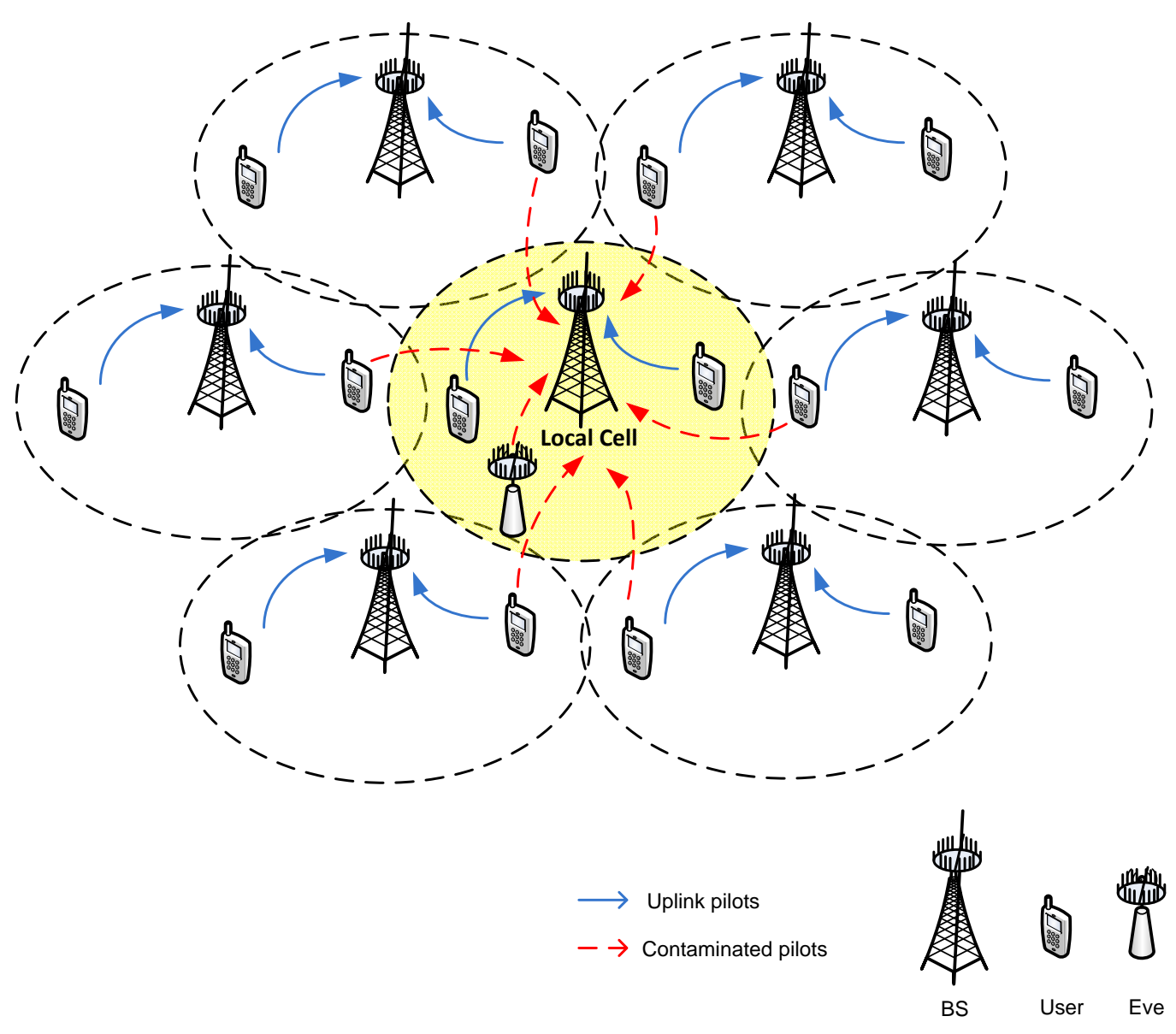

Fig. 1: Model of considered multi-cell massive MIMO system.

matrix $\widehat{\mathbf{R}}_{0 m}^{0}$ is given by

$$
\begin{gathered}
\widehat{\mathbf{R}}_{0 m}^{0}=P_{0 m} \tau \mathbf{R}_{0 m}^{0} \\
\times\left(N_{0} \mathbf{I}_{N_{t}}+\tau\left(\sum_{t=0}^{L} P_{t m} \mathbf{R}_{t m}^{0}+P_{E} r_{E, R}^{0} \mathbf{R}_{E, T}^{0}\right)\right)^{-1} \mathbf{R}_{0 m}^{0}
\end{gathered}
$$

Similarly, the MMSE channel estimates for the $m$ th user and the $k$ th user, $k=1,2, \cdots, K, k \neq m$, in the $l$ th cell, $l=$ $0,1, \cdots, L$, are given by

$$
\begin{aligned}
& \widehat{\mathbf{h}}_{l m}^{l}=\sqrt{P_{l m}} \mathbf{R}_{l m}^{l} \\
& \times\left(N_{0} \mathbf{I}_{N_{t}}+\tau\left(\sum_{t=0}^{L} P_{t k} \mathbf{R}_{t m}^{l}+P_{E} r_{E, R}^{l} \mathbf{R}_{E, T}^{l}\right)\right)^{-1} \widetilde{\mathbf{y}}_{l m},
\end{aligned}
$$

$$
\begin{aligned}
\tilde{\mathbf{y}}_{l m}=\sqrt{P_{l m}} \tau \mathbf{h}_{l m}^{l}+\sum_{t=0, t \neq l}^{L} \sqrt{P_{t m}} \tau \mathbf{h}_{t m}^{l} \\
+\sqrt{\frac{P_{E}}{N_{e}}} \tau \sum_{r=1}^{N_{e}} \mathbf{h}_{E, r}^{l}+\left(\boldsymbol{\omega}_{m} \otimes \mathbf{I}_{N_{t}}\right)^{H} \mathbf{n}
\end{aligned}
$$

and

$$
\begin{aligned}
& \widehat{\mathbf{h}}_{l k}^{l}=\sqrt{P_{l k}} \mathbf{R}_{l k}^{l}\left(N_{0} \mathbf{I}_{N_{t}}+\tau \sum_{t=0}^{L} P_{t k} \mathbf{R}_{t k}^{l}\right)^{-1} \widetilde{\mathbf{y}}_{l k} \\
& \widetilde{\mathbf{y}}_{l k}=\sqrt{P_{l k}} \tau \mathbf{h}_{l k}^{l}+\sum_{t=0, t \neq l}^{L} \sqrt{P_{t m}} \tau \mathbf{h}_{t k}^{l}+\left(\boldsymbol{\omega}_{k} \otimes \mathbf{I}_{N_{t}}\right)^{H} \mathbf{n}
\end{aligned}
$$

respectively. Furthermore, the correlation matrices of $\widehat{\mathbf{h}}_{l m}^{l}$ and $\widehat{\mathbf{h}}_{l k}^{l}$ are obtained as

$$
\begin{aligned}
& \widehat{\mathbf{R}}_{l m}^{l}=P_{l m} \tau \mathbf{R}_{l m}^{l} \\
& \times\left(N_{0} \mathbf{I}_{N_{t}}+\tau\left(\sum_{t=0}^{L} P_{t k} \mathbf{R}_{t m}^{l}+P_{E} r_{E, R}^{l} \mathbf{R}_{E, T}^{l}\right)\right)^{-1} \mathbf{R}_{l m}^{l}
\end{aligned}
$$




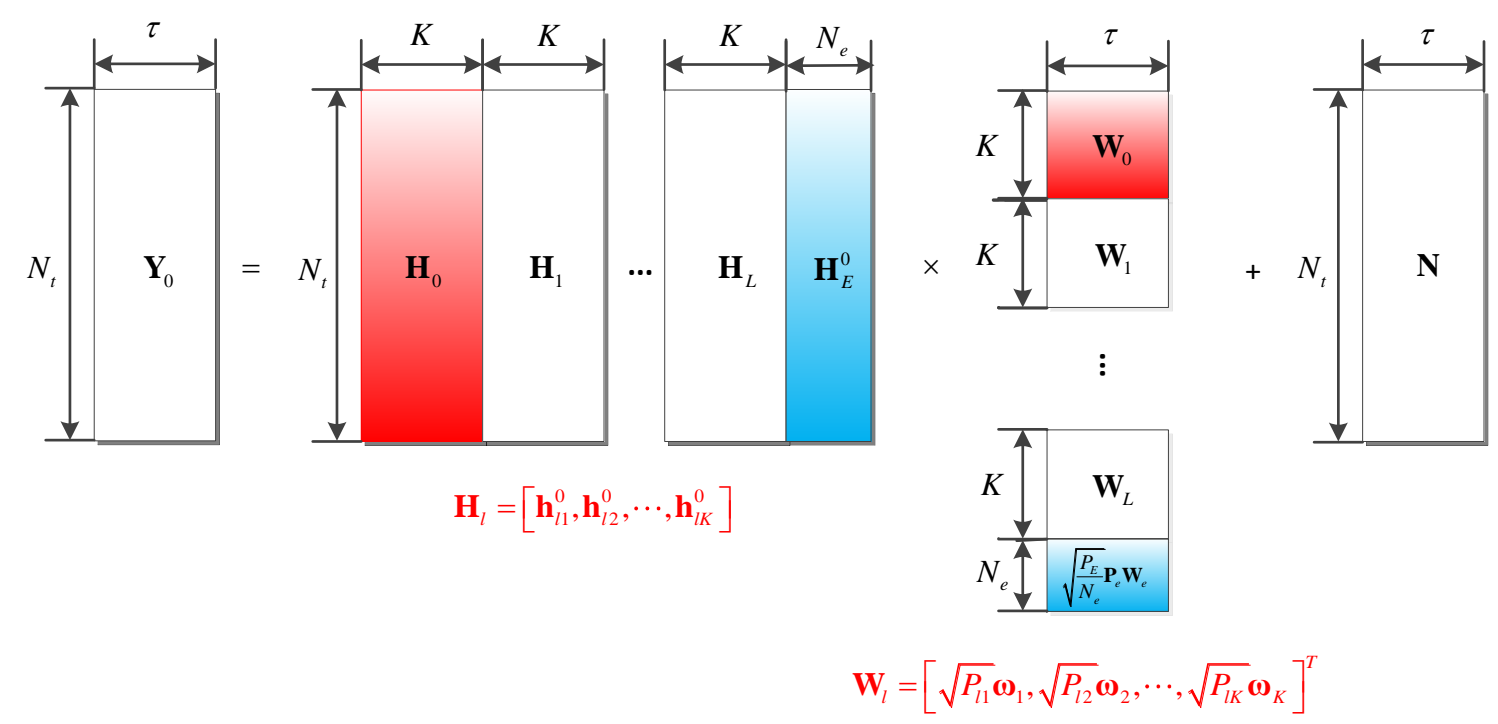

Fig. 2: Structure of uplink received signal at the BS in the local cell.

and

$$
\widehat{\mathbf{R}}_{l k}^{l}=P_{l k} \tau \mathbf{R}_{l k}^{l}\left(N_{0} \mathbf{I}_{N_{t}}+\tau \sum_{t=0}^{L} P_{t k} \mathbf{R}_{t k}^{l}\right)^{-1} \mathbf{R}_{l k}^{l},
$$

respectively.

Remark 1: We assume that the correlation matrices $\mathbf{R}_{t m}^{l}$ of the users and $r_{E, R}^{l} \mathbf{R}_{E, T}^{l}$ of the eavesdropper are perfectly known at the legitimate transmitter, see (3), (6), (8). In this context, we note that for massive MIMO systems, it is reasonable to assume that the statistical CSI of the users of the system is known at the BS $[3,47]$. Hence, our system model is applicable to the case where the BS attempts to transmit a private message to some users while treating the other users as eavesdroppers, i.e., the eavesdropper is an idle user of the system. Therefore, the statistical CSI of the eavesdropper can be assumed to be known. Nevertheless, the assumption that the statistical CSI of the active eavesdropper is available at the transmitter may also be reasonable if the eavesdropper is not an idle user. In particular, we can obtain $E\left[\widetilde{\mathbf{y}}_{l m} \widetilde{\mathbf{y}}_{l m}^{H}\right]$ by averaging $\widetilde{\mathbf{y}}_{l m}$ over different data slots. Eq. (7) suggests that $E\left[\widetilde{\mathbf{y}}_{l m} \widetilde{\mathbf{y}}_{l m}^{H}\right]$ is the sum of the correlation matrices of all users, the eavesdropper, and the noise. Then, $r_{E, R}^{l} \mathbf{R}_{E, T}^{l}$ can be obtained by subtracting the correlation matrices of the legitimate users and the noise from $E\left[\widetilde{\mathbf{y}}_{l m} \widetilde{\mathbf{y}}_{l m}^{H}\right]$.

Remark 2: For conventional massive MIMO systems with pilot contamination but without active eavesdropping [1,2], the term $P_{E} r_{E, R}^{0} \mathbf{R}_{E, T}^{0}$ is not present in (3). In this case, the BS can employ a user scheduling scheme [3,6] to control the pilot contamination and reduce the impact of $P_{t m} \mathbf{R}_{t m}^{0}$ in (3). However, this is not possible for active eavesdropping and the term $P_{E} r_{E, R}^{0} \mathbf{R}_{E, T}^{0}$ in (3) can not be avoided. This is an important difference between conventional massive MIMO systems and the system considered in this paper.
The eavesdropper can optimize its pilot contamination precoder $\mathbf{P}_{e}$ to conduct a best possible attack. Since the precoder is computed based on the estimated channel, the leakage of the desired signal will increase when the channel estimation error increases. Therefore, from the eavesdropper's perspective, the pilot contamination attack should impair the accuracy of the channel estimation as much as possible. As a result, the eavesdropper should optimize $\mathbf{P}_{e}$ such that the total average estimation error $\operatorname{tr}\left(\mathbf{R}_{0 m}^{0}-\widehat{\mathbf{R}}_{0 m}^{0}\right)$ is maximized. From (5), we observe that the total average estimation error $\operatorname{tr}\left(\mathbf{R}_{0 m}^{0}-\widehat{\mathbf{R}}_{0 m}^{0}\right)$ is a monotonically increasing function of $r_{E, R}^{0}$. Hence, maximizing $r_{E, R}^{0}$ is desirable. Therefore, finding the pilot contamination precoder $\mathbf{P}_{e}$ amounts to solving the following optimization problem

$$
\begin{gathered}
\max _{\mathbf{P}_{e}} \sum_{r=1}^{N_{e}} \sum_{s=1}^{N_{e}}\left\{\mathbf{P}_{e}^{H} \mathbf{R}_{E, R}^{0} \mathbf{P}_{e}\right\}_{r s} \\
\text { s.t. } \operatorname{tr}\left(\mathbf{P}_{e} \mathbf{W}_{e} \mathbf{W}_{e}^{H} \mathbf{P}_{e}^{H}\right) \leq N_{e} \tau .
\end{gathered}
$$

Let $\mathbf{P}_{e}=\left[\mathbf{p}_{1}, \mathbf{p}_{2}, \cdots, \mathbf{p}_{N_{e}}\right]$, where $\mathbf{p}_{s} \in \mathbb{C}^{N_{e} \times 1}$ is the $s$ th column of $\mathbf{P}_{e}$. Then, we have the following theorem.

Theorem 1: The optimal pilot contamination precoder $\mathbf{P}_{e}$ which solves (12) has to satisfy the following condition

$$
\sum_{s=1}^{N_{e}} \mathbf{p}_{s}=\sqrt{N_{e}} \mathbf{u}_{e}
$$

where $\mathbf{u}_{e} \in \mathbb{C}^{N_{e} \times 1}$ is the eigenvector corresponding to the largest eigenvalue of $\mathbf{R}_{E, R}^{0}$.

Proof: Please refer to Appendix A.

Theorem 1 indicates that transmitting the pilot sequence along the direction of the eigenvector corresponding to the maximum eigenvalue of the receive correlation matrix of the 
eavesdropper's channel constitutes the best possible attack strategy from the eavesdropper's point of view.

\section{B. Downlink Data Transmission}

For the data transmission phase, we assume that the BSs in all $L+1$ cells perform jamming to prevent eavesdropping in their own cells. Then, the transmit signal in the lth cell, $l=0,1, \cdots, L$, is given by

$$
\mathbf{x}_{l}=\sqrt{P}\left(\sqrt{p} \sum_{k=1}^{K} \mathbf{w}_{l k} s_{l k}+\sqrt{q} \mathbf{U}_{\text {null }, l} \mathbf{z}_{l}\right),
$$

where $P$ is the average transmit power for downlink transmission, $s_{l k}$ is the transmit signal for the $k$ th user in the $l$ th cell with $E\left[\left|s_{l k}\right|^{2}\right]=1$, and $p$ and $q$ denote the fractions of power allocated to transmit signal and AN, respectively. To avoid the high implementation complexity associated with the matrix inversion required for zero forcing and MMSE precoding [48], in this paper, we adopt simple matched filter precoding, as is typical for massive MIMO systems [1,2, 40]. Thus, we set $\mathbf{w}_{l k}=\frac{\widehat{\mathbf{h}}_{l k}^{l}}{\left\|\widehat{\mathbf{h}}_{l k}^{l}\right\|}$ for the precoding vector of the $k$ th user in the $l$ th cell. Furthermore, in (14), $\mathbf{U}_{\text {null, } l}$ and $\mathbf{z}_{l} \sim \mathcal{C N}\left(\mathbf{0}, \mathbf{I}_{N_{t}}\right)$ denote the AN shaping matrix and the AN vector in the $l$ th cell, respectively. We introduce $\widehat{\mathbf{H}}_{l}^{l}=\left[\widehat{\mathbf{h}}_{l 1}^{l}, \widehat{\mathbf{h}}_{l 2}^{l}, \cdots, \widehat{\mathbf{h}}_{l K}^{l}\right]$ for notational simplicity. If the AN shaping matrix is chosen as the NS of $\widehat{\mathbf{H}}_{l}^{l}$ as is conventionally done [33], for each new channel estimate, a matrix inversion is needed to compute the AN shaping matrix. This leads to a high implementation complexity considering the large numbers of antennas in massive MIMO. Thus, to keep the implementation complexity low despite the large numbers of antennas, we adopt for the AN shaping matrix the asymptotic NS of $\widehat{\mathbf{H}}_{l}^{l}$. In particular, since based on [52, Corollary 1] we have $\frac{1}{N_{t}}\left(\widehat{\mathbf{H}}_{l}^{l}\right)^{H} \widehat{\mathbf{H}}_{l}^{l} \stackrel{N_{t} \rightarrow \infty}{\rightarrow} \frac{1}{N_{t}} \operatorname{diag}\left[\operatorname{tr}\left(\widehat{\mathbf{R}}_{l 1}^{l}\right), \operatorname{tr}\left(\widehat{\mathbf{R}}_{l 2}^{l}\right), \cdots, \operatorname{tr}\left(\widehat{\mathbf{R}}_{l K}^{l}\right)\right]$, we set $\mathbf{U}_{\text {null }, l}=\mathbf{I}_{N_{t}}$ $\widehat{\mathbf{H}}_{l}^{l} \operatorname{diag}\left[\operatorname{tr}\left(\widehat{\mathbf{R}}_{l 1}^{l}\right)^{-1}, \operatorname{tr}\left(\widehat{\mathbf{R}}_{l 2}^{l}\right)^{-1}, \cdots, \operatorname{tr}\left(\widehat{\mathbf{R}}_{l K}^{l}\right)^{-1}\right]\left(\widehat{\mathbf{H}}_{l}^{l}\right)^{H}$. It can be shown that [52, Corollary 1]

$$
\frac{1}{N_{t}} \operatorname{tr}\left(\mathbf{U}_{\text {null }, l} \mathbf{U}_{\text {null }, l}^{H}\right) \stackrel{N_{t} \rightarrow \infty}{\rightarrow} \frac{1}{N_{t}}\left(N_{t}-K\right) .
$$

Based on (14), (15), and the expression for $\mathbf{w}_{l k}$, we set $K p+$ $\left(N_{t}-K\right) q=1$ to ensure $\mathbf{x}_{l}^{H} \mathbf{x}_{l} \stackrel{N_{t} \rightarrow \infty}{\rightarrow} P$.

The received signals at the $m$ th user in the reference cell, $y_{0 m}$, and at the eavesdropper, $\mathbf{y}_{\text {eve }} \in \mathbb{C}^{N_{e} \times 1}$, are given by

$$
y_{0 m}=\sum_{l=0}^{L}\left(\mathbf{h}_{0 m}^{l}\right)^{H} \mathbf{x}_{l}+n_{0 m}
$$

and

$$
\mathbf{y}_{\text {eve }}=\sum_{l=0}^{L}\left(\mathbf{H}_{E}^{l}\right)^{H} \mathbf{x}_{l}+\mathbf{n}_{\text {eve }}
$$

respectively. Here, $n_{0 m}$ and $\mathbf{n}_{\text {eve }} \in \mathbb{C}^{N_{e} \times 1}$ are zero-mean Gaussian noise processes with variance $N_{0, \mathrm{~d}}$ and covariance matrix $N_{0, \mathrm{~d}} \mathbf{I}_{N_{e}}$, respectively. We define the signal-to-noise ratio (SNR) for downlink data transmission as $\gamma=P / N_{0, \mathrm{~d}}$.
The structure of the downlink received signal at the desired user and the eavesdropper is illustrated in Figure 3.

In order to illustrate the secrecy threat that the pilot contamination attack poses for massive MIMO systems, we simulate the secrecy rate for MF precoding without $\mathrm{AN}$ generation ${ }^{1}$ (i.e., $p=1, q=0)$ in the following example.

Example 1: Let $N_{t}=128, N_{e}=1, L=3, K=5$, $P_{l k}=1, \forall k, \forall l, P_{E}=0.5$, and $N_{0}=1$. The achievable ergodic secrecy rate [40] of a massive MIMO system with MF precoding without AN generation is shown in Table I for i.i.d. fading and different SNR values.

The results in Table I reveal that even if the pilot power of the eavesdropper is only half of the pilot power of the desired user and the eavesdropper has only a single antenna, zero secrecy rate may result.

\section{Massive Mimo Signal Design for Combating the Pilot Contamination ATtack}

In this section, we investigate three signal designs for combating the pilot contamination attack by an active eavesdropper in a multi-cell multi-user massive MIMO system.

\section{A. MF-AN Design}

An achievable ergodic secrecy rate of the massive MIMO system described in Section II is given by [40]

$$
R_{\mathrm{sec}}=\left[R_{0 m}-C_{\mathrm{eve}}\right]^{+},
$$

where $R_{0 m}$ and $C_{\text {eve }}$ denote an achievable ergodic rate between the BS and the $m$ th user and the ergodic capacity between the BS and the eavesdropper in the 0th cell, respectively. The achievable ergodic rate $R_{0 m}$ is given by [40, Eq. (8)]

$$
R_{0 m}=E\left[\log _{2}\left(1+\operatorname{SINR}_{0 m}\right)\right]
$$

where $\operatorname{SINR}_{0 m}$ is given by

$$
\operatorname{SINR}_{0 m}=\frac{p \gamma\left|\left(\mathbf{h}_{0 m}^{0}\right)^{H} \mathbf{w}_{0 m}\right|^{2}}{A}
$$

$$
\begin{gathered}
A=p \gamma \sum_{k=1, k \neq m}^{K}\left|\left(\mathbf{h}_{0 m}^{0}\right)^{H} \mathbf{w}_{0 k}\right|^{2}+q \gamma\left|\left(\mathbf{h}_{0 m}^{0}\right)^{H} \mathbf{U}_{\text {null }, 0}\right|^{2} \\
+p \gamma \sum_{l=1}^{L} \sum_{k=1}^{K}\left|\left(\mathbf{h}_{0 m}^{l}\right)^{H} \mathbf{w}_{l k}\right|^{2}+q \gamma \sum_{l=1}^{L}\left|\left(\mathbf{h}_{0 m}^{l}\right)^{H} \mathbf{U}_{\text {null }, l}\right|^{2}+1 .
\end{gathered}
$$

In this paper, we make the pessimistic assumption that the eavesdropper has perfect knowledge of its own channel and is able to decode and cancel the signals of all intracell and inter-cell users from the received signal $\mathbf{y}_{\text {eve }}$ in (17) except for the signal intended for the $m$ th user in the 0th cell. This assumption results in an upper bound on the eavesdropper's capacity, and consequently, in a lower bound on the ergodic secrecy rate. If the eavesdropper has access to the data of all intra-cell and inter-cell interfering users,

\footnotetext{
${ }^{1}$ MF precoding without AN generation is often adopted in massive MIMO systems without secrecy consideration $[1,2]$.
} 


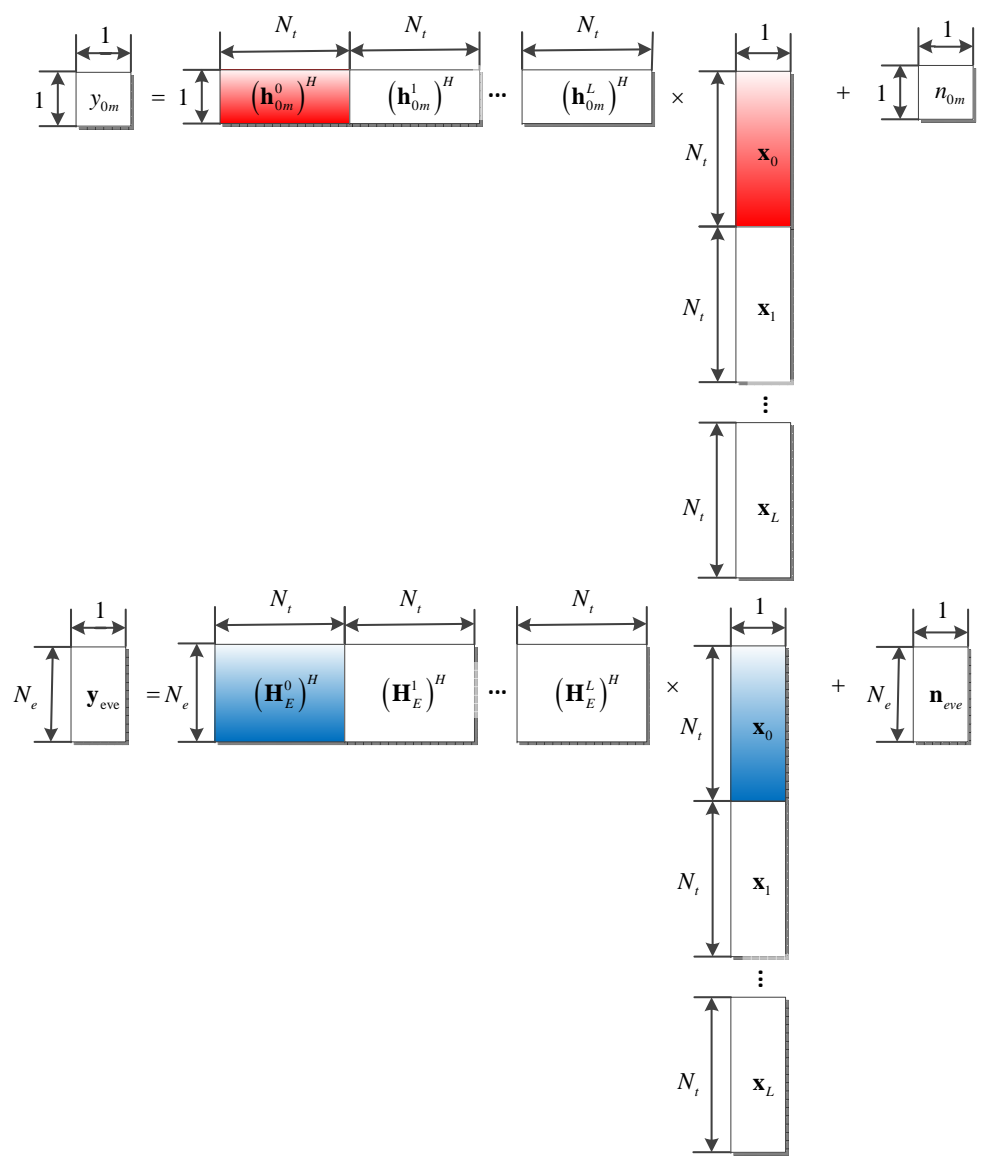

Fig. 3: Structure of the downlink received signal for the desired user and the eavesdropper.

TABLE I: The achievable secrecy rate for different SNR values.

\begin{tabular}{|c|c|c|c|c|c|c|}
\hline$\gamma(\mathrm{dB})$ & -10 & -8 & -6 & -4 & -2 & 0 \\
\hline Secrecy Rate $(\mathrm{b} / \mathrm{s} / \mathrm{Hz})$ & 0.3828 & 0.4097 & 0.3807 & 0.2759 & 0.0822 & 0 \\
\hline
\end{tabular}

this low bound is achievable. This might be the case if the interfering users cooperate with the eavesdropper. We note that this assumption constitutes a worst-case scenario. Hence, if secure communication can be achieved for this worst case, then secure communication can also be achieved for more optimistic settings (e.g. when the eavesdropper cannot mitigate all multi-user interference). Considering the worst case, $C_{\text {eve }}$ can be expressed as [40, Eq. (7)]

$$
\begin{aligned}
& C_{\text {eve }} \\
& =E\left[\log _{2}\left(1+p \gamma\left(\mathbf{w}_{0 m}\right)^{H} \mathbf{H}_{E}^{0} \mathbf{Q}^{-1}\left(\mathbf{H}_{E}^{0}\right)^{H} \mathbf{w}_{0 m}\right)\right] \\
& =E\left[\log _{2}\left(1+\frac{p \gamma}{\left\|\widehat{\mathbf{h}}_{0 m}^{0}\right\|^{2}}\left(\widehat{\mathbf{h}}_{0 m}^{0}\right)^{H} \mathbf{H}_{E}^{0} \mathbf{Q}^{-1}\left(\mathbf{H}_{E}^{0}\right)^{H} \widehat{\mathbf{h}}_{0 m}^{0}\right)\right],
\end{aligned}
$$

where

$$
\mathbf{Q}=q \gamma \sum_{l=0}^{L}\left(\mathbf{H}_{E}^{l}\right)^{H} \mathbf{U}_{\text {null }, l} \mathbf{U}_{\text {null }, l}^{H} \mathbf{H}_{E}^{l}+\mathbf{I}_{N_{e}}
$$

denotes the noise correlation matrix at the eavesdropper.

In the following theorem, we provide an asymptotic achievable secrecy rate expression when the number of transmit antennas $N_{t}$ tends to infinity. For convenience, the notation used in the theorem is summarized in Table II.

Theorem 2: An asymptotic achievable secrecy rate for a multi-cell multi-user massive MIMO system employing the MF-AN design to overcome a multi-antenna active eavesdropper is given by

$$
\begin{aligned}
R_{\text {sec, asy }} \stackrel{N_{t} \rightarrow \infty}{\rightarrow}\left[\log _{2}(1\right. & \left.+\operatorname{SINR}_{0 m, \text { asy }}\right) \\
& \left.-\log _{2}\left(1+\text { SINR }_{\text {eve }, \text { asy }}\right)\right]^{+}
\end{aligned}
$$


TABLE II: Notation used in Theorem 2

\begin{tabular}{|c|c|}
\hline Notation & Output SINR of the desired user in the asymptotic regime $N_{t} \rightarrow \infty$ \\
\hline SINR $_{0 m, \text { asy }}$ & Output SINR of the eavesdropper in the asymptotic regime $N_{t} \rightarrow \infty$ \\
\hline SINR $_{\text {eve, asy }}$ & Power of signal intended for the $m$ th user in the 0 th cell received by the desired user \\
\hline$\theta_{m}$ & Power of multi-user interference at the desired user \\
\hline$\theta_{b, p}$ & Power of the AN at the desired user \\
\hline$\theta_{b, q}$ & Power of the signal intended for the $m$ th user in the $l$ th cell received at the desired user \\
\hline$\Lambda_{0 m}^{l}$ & Power of AN received at the eavesdropper \\
\hline $\mathbf{Q}_{\text {asy }}$ & Power of the signal intended for the $m$ th user in the 0 th cell received at the eavesdropper \\
\hline$\eta_{i j}^{0}$ & Power of the signal intended for the $m$ th user in the $l$ th cell received at the eavesdropper \\
\hline$\eta_{i j}^{l}$ &
\end{tabular}

where

$$
\begin{aligned}
\mathrm{SINR}_{0 m, \text { asy }} & =\frac{p \gamma \theta_{m}}{p \gamma \theta_{b, p}+q \gamma \theta_{b, q}+1}, \\
\mathrm{SINR}_{\text {eve, asy }} & =\frac{p \gamma \theta_{e}}{\operatorname{tr}\left(\widehat{\mathbf{R}}_{0 m}^{0}\right)},
\end{aligned}
$$

with

$$
\begin{aligned}
\theta_{m}=\operatorname{tr}\left(\widehat{\mathbf{R}}_{0 m}^{0}\right)+\operatorname{tr}\left(\widehat{\mathbf{R}}_{0 m}^{0}\right)^{-1} \operatorname{tr}\left(\left(\mathbf{R}_{0 m}^{0}-\widehat{\mathbf{R}}_{0 m}^{0}\right) \widehat{\mathbf{R}}_{0 m}^{0}\right)_{(27)} \\
\theta_{b, p}=\sum_{l=0}^{L} \sum_{k=1, k \neq m}^{K} \operatorname{tr}\left(\widehat{\mathbf{R}}_{l k}^{l}\right)^{-1} \operatorname{tr}\left(\mathbf{R}_{0 m}^{l} \widehat{\mathbf{R}}_{l k}^{l}\right) \\
+\sum_{l=1}^{L} \operatorname{tr}\left(\widehat{\mathbf{R}}_{l m}^{l}\right)^{-1} \Lambda_{0 m}^{l} \quad(28) \\
\theta_{b, q}=\sum_{l=0}^{L} \operatorname{tr}\left(\mathbf{R}_{0 m}^{l}\right)-\sum_{l=0}^{L} \sum_{k=1, k \neq m}^{K} \operatorname{tr}\left(\widehat{\mathbf{R}}_{l k}^{l}\right)^{-1} \operatorname{tr}\left(\mathbf{R}_{0 m}^{l} \widehat{\mathbf{R}}_{l k}^{l}\right) \\
-\sum_{l=0}^{L} \operatorname{tr}\left(\widehat{\mathbf{R}}_{l m}^{l}\right)^{-1} \Lambda_{0 m}^{l} . \quad(29)
\end{aligned}
$$

Here, $\Lambda_{0 m}^{l}$ in (28) and (29) is given by

$$
\begin{aligned}
\Lambda_{0 m}^{l}= & \tau^{2} P_{0 m}\left|\operatorname{tr}\left(\mathbf{C}_{l m}^{l} \mathbf{R}_{0 m}^{l}\right)\right|^{2}+\tau N_{0} \operatorname{tr}\left(\mathbf{R}_{0 m}^{l} \mathbf{C}_{l m}^{l}\left(\mathbf{C}_{l m}^{l}\right)^{H}\right) \\
& +\tau^{2} \sum_{t=1}^{L} P_{t m} \operatorname{tr}\left(\mathbf{R}_{0 m}^{l} \mathbf{C}_{l m}^{l} \mathbf{R}_{t m}^{l}\left(\mathbf{C}_{l m}^{l}\right)^{H}\right) \\
& +\tau^{2} P_{E} r_{E, R}^{0} \operatorname{tr}\left(\mathbf{R}_{0 m}^{l} \mathbf{C}_{l m}^{l} \mathbf{R}_{E, T}^{l}\left(\mathbf{C}_{l m}^{l}\right)^{H}\right)
\end{aligned}
$$

$$
\begin{aligned}
& \mathbf{C}_{l m}^{l}=\sqrt{P_{l m}} \mathbf{R}_{l m}^{l} \\
& \times\left(N_{0} \mathbf{I}_{N_{t}}+\tau\left(\sum_{t=0}^{L} P_{t m} \mathbf{R}_{t m}^{l}+P_{E} r_{E, R}^{l} \mathbf{R}_{E, T}^{l}\right)\right)^{-1} .
\end{aligned}
$$

Furthermore, $\theta_{e}$ in (26) is given by

$$
\theta_{e}=\sum_{i=1}^{N_{e}} \sum_{j=1}^{N_{e}}\left\{\mathbf{Q}_{\mathrm{asy}}^{-1}\right\}_{i j} \eta_{i j}^{0},
$$

where

$$
\begin{gathered}
\eta_{i j}^{l}=\tau^{2}\left\{\mathbf{R}_{E, R}^{l}\right\}_{i j} \sum_{t=0}^{L} P_{t m} \operatorname{tr}\left(\mathbf{R}_{E, T}^{l} \mathbf{C}_{l m}^{l} \mathbf{R}_{t m}^{l}\left(\mathbf{C}_{l m}^{l}\right)^{H}\right) \\
+\tau^{2} \frac{P_{E}}{N_{e}} \sum_{r=1}^{N_{e}}\left\{\mathbf{R}_{E, R}^{l}\right\}_{i r} \sum_{r=1}^{N_{e}}\left\{\mathbf{R}_{E, R}^{l}\right\}_{r j}\left|\operatorname{tr}\left(\mathbf{C}_{l m}^{l} \mathbf{R}_{E, T}^{l}\right)\right|^{2} \\
+N_{0} \tau\left\{\mathbf{R}_{E, R}^{l}\right\}_{i j} \operatorname{tr}\left(\mathbf{R}_{E, T}^{l} \mathbf{C}_{l m}^{l}\left(\mathbf{C}_{l m}^{l}\right)^{H}\right)
\end{gathered}
$$

and $\mathbf{Q}_{\text {asy }}=q \gamma \sum_{l=0}^{L} \mathbf{Q}_{l}+\mathbf{I}_{N_{r}}$. Matrix $\mathbf{Q}_{l} \in \mathbb{C}^{N_{e} \times N_{e}}$ has elements

$$
\begin{aligned}
& \left\{\mathbf{Q}_{l}\right\}_{i j}=\left\{\mathbf{R}_{E, R}^{l}\right\}_{i j} \operatorname{tr}\left(\mathbf{R}_{E, T}^{l}\right)-\left[\left\{\mathbf{R}_{E, R}^{l}\right\}_{i j}\right. \\
& \left.\times \sum_{k=1, k \neq m}^{K} \operatorname{tr}\left(\widehat{\mathbf{R}}_{l k}^{l}\right)^{-1} \operatorname{tr}\left(\mathbf{R}_{E, T}^{l} \widehat{\mathbf{R}}_{l k}^{l}\right)+\operatorname{tr}\left(\widehat{\mathbf{R}}_{l m}^{l}\right)^{-1} \eta_{i j}^{l}\right] .
\end{aligned}
$$

Proof: Please refer to Appendix B.

Theorem 2 provides an expression for the general asymptotic achievable secrecy rate for $N_{t} \rightarrow \infty$, which is valid for arbitrary $p$ and $q$. In practice, the optimal $p$ can be found by performing a simple one dimensional numerical search in the interval $0 \leq p \leq \frac{1}{K}$ for maximization of (24). For the special case of a single-antenna eavesdropper, the optimal $p$ can be obtained in closed form, c.f. Section IV-A.

For the conventional secrecy problem existing in multipleinput, single-output systems with multiple-antenna passive eavesdroppers [24], the optimal transmission design does not require $\mathrm{AN}$ generation and the secrecy rate increases for increasing SNR. To illustrate the importance of AN generation when an active eavesdropper is present, we introduce the following theorem.

Theorem 3: For the case without AN generation where $p=$ $\frac{1}{K}, R_{\text {sec, asy }}$ in (24) is a monotonically decreasing function of $\gamma$ for $\gamma>\gamma_{\mathrm{th}}$, where

$$
\gamma_{\mathrm{th}}=\frac{-\theta_{b, p} \tilde{\theta}_{e}+\sqrt{\theta_{b, p}^{2} \tilde{\theta}_{e}^{2}+\left(\theta_{b, p}+\theta_{m}\right)\left(\theta_{m}-\tilde{\theta}_{e}\right) \theta_{b, p} \tilde{\theta}_{e}}}{\left(\theta_{b, p}+\theta_{m}\right) \theta_{b, p} \tilde{\theta}_{e}}
$$

with

$$
\tilde{\theta}_{e}=\frac{\sum_{i=1}^{N_{e}} \eta_{i i}^{0}}{\operatorname{tr}\left(\widehat{\mathbf{R}}_{0 m}^{0}\right)}
$$


Proof: Please refer to Appendix C.

Remark 3: Theorem 3 indicates that in the presence of an active eavesdropper, the secrecy rate will decrease for increasing SNR in the high SNR regime if all the available power at the BS is allocated to the information-carrying signals. This behaviour is very different from that of multipleantenna systems with passive eavesdroppers [24], where the secrecy rate is a monotonically increasing function of the SNR throughout the entire SNR region even if AN is not employed. However, for the case considered in this paper, the BS relies on estimated CSI for precoder design, which is contaminated by the attack of the active eavesdropper. Since the BS cannot distinguish the actual channel of the desired user from the eavesdropper channel, the precoder may implicitly beamform the information signal towards the eavesdropper. As a result, the capacity of the eavesdropper may increase faster than the achievable rate of the desired user as the transmit power increases. Hence, it is advantageous if a fraction of power is used to generate AN to degrade the eavesdropper's ability to decode the transmit signal intended for the desired user. This is the motivation behind the MF-AN design.

\section{B. NS Design}

In order to obtain some insight for transmit signal design, in the following theorem, we investigate the asymptotic achievable secrecy rate under the assumption that the transmit correlation matrices of the users are orthogonal to the transmit correlation matrices of the eavesdropper. The notations used in the following theorem are summarized in Table III, where the subscript "orth" in the variable names indicates that the transmit correlation matrices of the users are assumed to be orthogonal to the transmit correlation matrices of the eavesdropper.

Theorem 4: If $\sum_{t=0}^{L} \operatorname{tr}\left(\mathbf{R}_{t m}^{l} \mathbf{R}_{E, T}^{l}\right)=0$ for $l=$ $0,1, \cdots, L$, the secrecy rate $R_{\mathrm{sec} \text {, asy }}$ is identical to the achievable rate of the desired user $R_{\text {sec, asy, orth }}=\log _{2}(1+$ $\mathrm{SINR}_{0 m \text {, asy, orth }}$, where

$$
\mathrm{SINR}_{0 m, \text { asy }, \text { orth }}=\frac{p \gamma \theta_{m, \text { orth }}}{p \gamma \theta_{b, p, \text { orth }}+q \gamma \theta_{b, q, \text { orth }}+1}
$$

with

$$
\begin{array}{r}
\theta_{m, \text { orth }}=\operatorname{tr}\left(\widehat{\mathbf{R}}_{0 m, \text { orth }}^{0}\right) \\
+\operatorname{tr}\left(\widehat{\mathbf{R}}_{0 m, \text { orth }}^{0}\right)^{-1} \operatorname{tr}\left(\left(\mathbf{R}_{0 m}^{0}-\widehat{\mathbf{R}}_{0 m, \text { orth }}^{0}\right) \widehat{\mathbf{R}}_{0 m, \text { orth }}^{0}\right) \\
\theta_{b, p, \text { orth }}=\sum_{l=0}^{L} \sum_{k=1, k \neq m}^{K} \operatorname{tr}\left(\widehat{\mathbf{R}}_{l k}^{l}\right)^{-1} \operatorname{tr}\left(\mathbf{R}_{0 m}^{l} \widehat{\mathbf{R}}_{l k}^{l}\right) \\
+\sum_{l=1}^{L} \operatorname{tr}\left(\widehat{\mathbf{R}}_{l m, \text { orth }}^{l}\right)^{-1} \Lambda_{0 m, \text { orth }}^{l}
\end{array}
$$

$$
\begin{gathered}
\theta_{b, q, \text { orth }}=\sum_{l=0}^{L} \operatorname{tr}\left(\mathbf{R}_{0 m}^{l}\right) \\
-\sum_{l=0}^{L} \sum_{k=1, k \neq m}^{K} \operatorname{tr}\left(\widehat{\mathbf{R}}_{l k}^{l}\right)^{-1} \operatorname{tr}\left(\mathbf{R}_{0 m}^{l} \widehat{\mathbf{R}}_{l k}^{l}\right) \\
-\sum_{l=0}^{L} \operatorname{tr}\left(\widehat{\mathbf{R}}_{l m, \text { orth }}^{l}\right)^{-1} \Lambda_{0 m, \text { orth }}^{l} \\
+\tau^{2} \sum_{t=1}^{L} P_{t m} \operatorname{tr}\left(\mathbf{R}_{0 m}^{l} \mathbf{C}_{l m, \text { orth }}^{l} \mathbf{R}_{t m}^{l}\left(\mathbf{C}_{l m, \text { orth }}^{l}\right)^{H}\right) \\
+\tau N_{0} \operatorname{tr}\left(\mathbf{R}_{0 m}^{l} \mathbf{C}_{l m, \text { orth }}^{l}\left(\mathbf{C}_{l m, \text { orth }}^{l}\right)^{H}\right) \\
\mathbf{C}_{l m, \text { orth }}^{l}=\sqrt{P_{l m}} \mathbf{R}_{l m}^{l}\left(N_{0} \mathbf{I}_{N_{t}}+\tau \sum_{t=0}^{L} P_{t m} \mathbf{R}_{t m}^{l}\right)^{-1}
\end{gathered}
$$

$$
\widehat{\mathbf{R}}_{l m, \text { orth }}^{l}=P_{l m} \tau \mathbf{R}_{l m}^{l}\left(N_{0} \mathbf{I}_{N_{t}}+\tau \sum_{t=0}^{L} P_{t m} \mathbf{R}_{t m}^{l}\right)^{-1} \mathbf{R}_{l m}^{l}
$$

Proof: Please refer to Appendix D.

Theorem 4 reveals that when the channels of the eavesdropper and the users are statistically orthogonal, the pilot contamination attack has no impact on the secrecy rate. It is known that for typical massive MIMO scenarios, the transmit correlation matrices of the channels are low rank $[3,4,6-8$, $10,38]$. Hence, inspired by Theorem 4 , in the remainder of this subsection, we introduce an NS based design where the information-carrying signal is transmitted in the NS of the transmit correlation matrix of the eavesdropper's channel $\mathbf{H}_{E}^{l}$.

Assume the rank of $\mathbf{R}_{E, T}^{l}$ is $T_{l}, l=0,1, \cdots, L$. Let us construct a matrix $\mathbf{V}_{E}^{l} \in \mathbb{C}^{N_{t} \times M_{l}}$, whose $M_{l}$ columns are the $M_{l}$ eigenvectors corresponding to the zero eigenvalues of $\mathbf{R}_{E, T}^{l}$, where $M_{l}=N_{t}-T_{l}$. In the uplink training phase, we multiply $\mathbf{Y}_{l}$ with $\mathbf{V}_{E}^{l}$ to obtain ${ }^{2}$

$$
\begin{aligned}
\left(\mathbf{V}_{E}^{l}\right)^{H} \mathbf{Y}_{l} & =\sum_{k=1}^{K} \sqrt{P_{l k}}\left(\mathbf{V}_{E}^{l}\right)^{H} \mathbf{h}_{l k}^{l} \boldsymbol{\omega}_{k}^{T} \\
& +\sum_{l=1}^{L} \sum_{k=1}^{K} \sqrt{P_{l k}}\left(\mathbf{V}_{E}^{l}\right)^{H} \mathbf{h}_{l k}^{l} \boldsymbol{\omega}_{k}^{T} \\
& +\sqrt{P_{E}}\left(\mathbf{V}_{E}^{l}\right)^{H} \mathbf{H}_{E}^{l} \mathbf{P}_{e} \mathbf{W}_{e}+\left(\mathbf{V}_{E}^{l}\right)^{H} \mathbf{N}
\end{aligned}
$$

\footnotetext{
${ }^{2}$ The $m$ th user in the $l$ th cell $(l=1,2, \cdots, L)$ is also affected by the pilot sequence sent by the active eavesdropper in the reference cell. As a result, we assume that the $l$ th BS also employs the NS design to combat the pilot contamination attack.
} 
TABLE III: Notation used in Theorem 4

\begin{tabular}{|c|c|}
\hline Notation & Description \\
\hline SINR $_{0 m, \text { asy }, \text { orth }}$ & Output SINR of the desired user in the asymptotic regime $N_{t} \rightarrow \infty$ \\
\hline$\theta_{m, \text { orth }}$ & Power of signal intended for the $m$ th user in the 0th cell received by the desired user \\
\hline$\theta_{b, p, \text { orth }}$ & Power of multi-user interference at the desired user \\
\hline$\theta_{b, q, \text { orth }}$ & Power of the AN received at the desired user \\
\hline$\Lambda_{0 m, \text { orth }}^{l}$ & Power of signal intended for the $m$ th user in the $l$ th cell received at the desired user \\
\hline
\end{tabular}

and

$$
\begin{aligned}
\mathbf{y}_{l, \text { null }}= & \operatorname{vec}\left(\left(\mathbf{V}_{E}^{l}\right)^{H} \mathbf{Y}_{l}\right)=\sum_{k=1}^{K} \sqrt{P_{l k}}\left(\boldsymbol{\omega}_{k} \otimes \mathbf{I}_{N_{t}}\right) \mathbf{h}_{l k, \text { null }}^{l} \\
& +\sum_{l=1}^{L} \sum_{k=1}^{K} \sqrt{P_{l k}}\left(\boldsymbol{\omega}_{k} \otimes \mathbf{I}_{N_{t}}\right) \mathbf{h}_{l k, \text { null }}^{l} \\
& +\sqrt{\frac{P_{E}}{N_{e}}}\left(\boldsymbol{\omega}_{m} \otimes \mathbf{I}_{N_{t}}\right) \sum_{r=1}^{N_{e}} \mathbf{h}_{\text {eff }, r, \text { null }}^{l}+\widetilde{\mathbf{n}}_{\text {null }}^{l}, \quad \text { (45) }
\end{aligned}
$$

where $\mathbf{h}_{l k, \text { null }}^{l}=\left(\mathbf{V}_{E}^{l}\right)^{H} \mathbf{h}_{l k}^{l} \sim \mathcal{C N}\left(\mathbf{0}, \mathbf{R}_{l k, \text { null }}^{l}\right), \mathbf{R}_{l k, \text { null }}^{l}=$ $\left(\mathbf{V}_{E}^{l}\right)^{H} \mathbf{R}_{l k}^{l} \mathbf{V}_{E}^{l} \in \mathbb{C}^{M_{l} \times M_{l}}, \quad l=0,1, \cdots, L, \quad k=$ $1,2, \cdots, K$, and $\widetilde{\mathbf{n}}_{\text {null }}^{l} \sim \mathcal{C N}\left(\mathbf{0}, \tau N_{0} \mathbf{I}_{M_{l}}\right) . \mathbf{h}_{\text {eff }, r, \text { null }}^{l}$ is the $r$ th column of matrix $\left(\mathbf{V}_{E}^{l}\right)^{H} \mathbf{H}_{E}^{l} \mathbf{P}_{e}$. We obtain an estimate for $\mathbf{h}_{l m, \text { null }}^{l}$ as

$$
\begin{aligned}
\widehat{\mathbf{h}}_{l m, \text { null }}^{l} & =\sqrt{P_{l m}} \mathbf{R}_{l m, \text { null }}^{l} \\
& \times\left(N_{0} \mathbf{I}_{M}+\tau \sum_{t=0}^{L} P_{t m} \mathbf{R}_{t m, \text { null }}^{l}\right)^{-1} \widetilde{\mathbf{y}}_{l m, \text { null }},
\end{aligned}
$$

where

$$
\begin{aligned}
\widetilde{\mathbf{y}}_{l m, \text { null }} & =\sqrt{P_{l m}} \tau \mathbf{h}_{l m, \text { null }}^{l}+\sum_{t=1}^{L} \sqrt{P_{t m}} \tau \mathbf{h}_{t m, \text { null }}^{l} \\
& +\sqrt{\frac{P_{E}}{N_{e}}} \sum_{r=1}^{N_{e}} \mathbf{h}_{\text {eff }, r, \text { null }}^{l}+\left(\boldsymbol{\omega}_{m} \otimes \mathbf{I}_{M}\right)^{H} \widetilde{\mathbf{n}}_{\text {null }}^{l} .
\end{aligned}
$$

The actual channel vector can be expressed as $\mathbf{h}_{l m, \text { null }}^{l}=$ $\widehat{\mathbf{h}}_{l m, \text { null }}^{l}+\mathbf{e}_{l m, \text { null }}^{l}$, where $\widehat{\mathbf{h}}_{l m}^{l} \sim \mathcal{C N}\left(\mathbf{0}, \widehat{\mathbf{R}}_{l m, \text { null }}^{l}\right)$ and $\mathbf{e}_{l m, \text { null }}^{l} \sim \mathcal{C N}\left(\mathbf{0}, \mathbf{R}_{l m, \text { null }}^{l}-\widehat{\mathbf{R}}_{l m, \text { null }}^{l}\right)$ with

$$
\begin{aligned}
\widehat{\mathbf{R}}_{l m, \text { null }}^{l} & =P_{l m} \tau \mathbf{R}_{l m, \text { null }}^{l} \\
& \times\left(N_{0} \mathbf{I}_{M}+\tau \sum_{t=0}^{L} P_{t m} \mathbf{R}_{t m, \text { null }}^{l}\right)^{-1} \mathbf{R}_{l m, \text { null }}^{l} .
\end{aligned}
$$

In the downlink data transmission phase, we employ the same transmission scheme as in Section II-B, but replace $\mathbf{w}_{l m}$ with $\mathbf{w}_{l m, \text { null }}=\mathbf{V}_{E}^{l} \frac{\widehat{\mathbf{h}}_{l m, \text { null }}^{l}}{\left\|\widehat{\mathbf{h}}_{l m, \text { null }}^{l}\right\|}$, and set $p=1 / K, q=0$.

Remark 4: The proposed NS design transmits the signal in the orthogonal subspace $\mathbf{V}_{E}^{0}$. As a result, the performance of the NS design depends on the rank of $\mathbf{V}_{E}^{0}$. For instance, for the extreme case of i.i.d. fading, $\mathbf{V}_{E}^{0}$ does not exist and hence the NS design is not applicable. In practice, the NS design will be beneficial in highly correlated channels, for a strong pilot contamination attack, and in the high SNR regime since it can effectively degrade the eavesdropper's performance in these scenarios. In contrast, we expect the MF-AN design to outperform the NS design in weakly correlated channels, for a weak pilot contamination attack, and in the low SNR regime.

\section{Unified Design}

Considering Remark 4, we propose a unified design that exploits the advantages of both the conventional MF-AN design and the NS design. The corresponding transmit signal in the $l$ th cell, $l=0,1, \cdots, L$, is given by

$$
\begin{aligned}
\mathbf{x}_{l}= & \sqrt{P}\left(\sqrt{\alpha}\left(\sqrt{p} \sum_{k=1}^{K} \mathbf{w}_{l k} s_{l k}+\sqrt{q} \mathbf{U}_{\text {null }, 1} \mathbf{z}_{l}\right)\right. \\
& \left.+\sqrt{\frac{\beta}{K}}\left(\mathbf{w}_{l m, \text { null }} s_{l k}+\sum_{k=1, k \neq m}^{K} \mathbf{w}_{l k} s_{l k}\right)\right),
\end{aligned}
$$

where $\alpha$ and $\beta$ denote the weights of the MF-AN design and the NS design with $\alpha+\beta=1$. Using the secrecy rate in (18) as the cost function, the optimal $\alpha$ and $\beta$ can be obtained from a one-dimensional numerical search.

\section{The Single-Antenna Eavesdropper CASE}

In this section, we analyze the performance of massive MIMO systems for the case of an active single-antenna eavesdropper [43-45]. For the MF-AN design, we derive closedform expressions for the optimal power allocation policy for the transmit signal and the $\mathrm{AN}$ as well as the minimum transmit signal power required to ensure secure transmission. Also, we derive a closed-form expression for a threshold that can be used to optimally switch between the MF-AN and the NS design. Finally, we investigate the single-cell single-user massive MIMO system.

\section{A. Multi-cell Multi-user Case}

We obtain a closed-form solution for the optimal power allocation for the MF-AN design in the following theorem. The notation used in the theorem is summarized in Table IV.

Theorem 5: Let us define

$$
\begin{aligned}
p_{1} & =-\frac{\left(a_{1} c_{2}-a_{2} c_{1}\right)}{a_{1} b_{2}-a_{2} b_{1}} \\
- & \frac{\sqrt{\left(a_{1} c_{2}-a_{2} c_{1}\right)^{2}-\left(a_{1} b_{2}-a_{2} b_{1}\right)\left(b_{1}-b_{2}\right) c_{1}}}{a_{1} b_{2}-a_{2} b_{1}}
\end{aligned}
$$


TABLE IV: Notations used in Theorem 5.

\begin{tabular}{|c|c|}
\hline Notation & Description \\
\hline$a_{1}, b_{1}, c_{1}$ & $a_{1} p^{2}+b_{1} p+c_{1}$ is the numerator of the ratio between $1+$ SINR $_{0 m, \text { asy }}$ and $1+$ SINR $_{\text {eve, asy }}$ when $N_{e}=1$ \\
\hline$a_{2}, b_{2}, c_{2}$ & $a_{2} p^{2}+b_{2} p+c_{2}$ is the denominator of the ratio between $1+$ SINR $_{0 m, \text { asy }}$ and $1+$ SINR $_{\text {eve, asy }}$ when $N_{e}=1$ \\
\hline$\theta_{e, e}$ & Power of the signal intended for the $m$ th user in the 0 th cell received at the eavesdropper when $N_{e}=1$ \\
\hline$\theta_{e, q}$ & Power of AN received at the eavesdropper when $N_{e}=1$ \\
\hline$\Lambda_{E}^{l}$ & Power of the signal intended for the $m$ th user in the $l$ th cell received at the eavesdropper when $N_{e}=1$ \\
\hline
\end{tabular}

and

$$
\begin{aligned}
p_{2} & =-\frac{\left(a_{1} c_{2}-a_{2} c_{1}\right)}{a_{1} b_{2}-a_{2} b_{1}} \\
+ & \frac{\sqrt{\left(a_{1} c_{2}-a_{2} c_{1}\right)^{2}-\left(a_{1} b_{2}-a_{2} b_{1}\right)\left(b_{1}-b_{2}\right) c_{1}}}{a_{1} b_{2}-a_{2} b_{1}},
\end{aligned}
$$

where

$$
\begin{gathered}
a_{1}=-\gamma^{2}\left(\left(N_{t}-K\right) \theta_{m}-\left(N_{t}-K\right) \theta_{b, p}-K \theta_{b, q}\right) K \theta_{e, q} \\
b_{1}=\gamma\left(\left(N_{t}-K\right) \theta_{m}+\left(N_{t}-K\right) \theta_{b, p}-K \theta_{b, q}\right) \\
\quad \times\left(\gamma \theta_{e, q}+N_{t}-K\right) \\
+\gamma\left(\gamma \theta_{b, q}+N_{t}-K\right)\left(\left(N_{t}-K\right) \theta_{e, q}-K \theta_{e, q}\right) \\
\quad c_{1}=\left(\gamma \theta_{b, q}+N_{t}-K\right)\left(\gamma \theta_{e, q}+N_{t}-K\right) \\
a_{2}=\gamma^{2}\left(\left(N_{t}-K\right) \theta_{b, p}-K \theta_{b, q}\right)\left(\left(N_{t}-K\right) \theta_{e, e}-K \theta_{e, q}\right)
\end{gathered}
$$

$$
\begin{gathered}
b_{2}=\gamma\left(\gamma \theta_{b, q}+N_{t}-K\right)\left(\left(N_{t}-K\right) \theta_{e, e}-K \theta_{e, q}\right) \\
+\gamma\left(\left(N_{t}-K\right) \theta_{b, p}-K \theta_{b, q}\right)\left(\gamma \theta_{e, q}+N_{t}-K\right) \\
c_{2}=\left(\gamma \theta_{b, q}+N_{t}-K\right)\left(\gamma \theta_{e, q}+N_{t}-K\right),
\end{gathered}
$$

with

$$
\begin{gathered}
\theta_{e, e}=\frac{\Lambda_{E}^{0}}{\operatorname{tr}\left(\widehat{\mathbf{R}}_{0 m}^{0}\right)}, \\
\theta_{e, q}=\sum_{l=0}^{L} \operatorname{tr}\left(\mathbf{R}_{E, T}^{l}\right)-\sum_{l=0}^{L} \sum_{k=1, k \neq m}^{K} \operatorname{tr}\left(\widehat{\mathbf{R}}_{l k}^{l}\right)^{-1} \operatorname{tr}\left(\mathbf{R}_{E, T}^{l} \widehat{\mathbf{R}}_{l k}^{l}\right) \\
-\sum_{l=0}^{L} \operatorname{tr}\left(\widehat{\mathbf{R}}_{l m}^{l}\right)^{-1} \Lambda_{E}^{l}
\end{gathered}
$$

and

$$
\begin{gathered}
\Lambda_{E}^{l}=\tau^{2} P_{E}\left|\operatorname{tr}\left(\mathbf{C}_{l m}^{l} \mathbf{R}_{E, T}^{l}\right)\right|^{2}+\tau N_{0} \operatorname{tr}\left(\mathbf{R}_{E, T}^{l} \mathbf{C}_{l m}^{l}\left(\mathbf{C}_{l m}^{l}\right)^{H}\right) \\
+\tau^{2} \sum_{t=0}^{L} P_{t m} \operatorname{tr}\left(\mathbf{R}_{E, T}^{l} \mathbf{C}_{l m}^{l} \mathbf{R}_{t m}^{l}\left(\mathbf{C}_{l m}^{l}\right)^{H}\right) .
\end{gathered}
$$

Then, the optimal power allocation $p$ maximizing the asymptotic achievable secrecy rate in (24) for $N_{e}=1$ is given in (61) at the top of the next page, where $R_{\mathrm{sec} \text {, asy }}(p)$ is defined in (24).
Proof: Please refer to Appendix E.

A fundamental question in secure communication is under which conditions a positive secrecy rate is achievable. For massive MIMO systems under the pilot contamination attack, this question is answered in the following theorem.

Theorem 6: For $N_{e}=1$, to achieve $R_{\mathrm{sec} \text {, asy }}>0$ in (24), the power allocated to the transmit signal must satisfy ${ }^{3}$ :

$$
\begin{aligned}
& p>-\frac{b_{1}-b_{2}}{a_{1}-a_{2}}, \text { if } a_{1}-a_{2}>0 \\
& p<-\frac{b_{1}-b_{2}}{a_{1}-a_{2}}, \text { if } a_{1}-a_{2}<0 .
\end{aligned}
$$

Proof: Eqs. (62) and (63) can be obtained by following a similar approach as in Appendix $\mathrm{E}$ and finding the solution to $\frac{1+\mathrm{SINR}_{0 m, \text { asy }}}{1+\mathrm{SINR}_{\text {eve, asy }}}>1$.

In the following, we derive a threshold which allows us to determine whether the MF-AN design or the NS design achieves a higher secrecy rate. Before proceeding, we introduce some definitions. Define $a_{3}=$ $q^{*} \theta_{e, q}\left(p^{*} \theta_{b, p}+q^{*} \theta_{b, q}+p^{*} \theta_{m}\right), b_{3}=q^{*} \theta_{e, q}+p^{*} \theta_{b, p}+$ $q^{*} \theta_{b, q}+p^{*} \theta_{m}, a_{4}=\left(p^{*} \theta_{b, p}+q^{*} \theta_{b, q}\right)\left(p^{*} \theta_{e, e}+q^{*} \theta_{e, q}\right), b_{4}=$ $p^{*} \theta_{b, p}+q^{*} \theta_{b, q}+p^{*} \theta_{e, e}+q^{*} \theta_{e, q}$, where $p^{*}$ and $q^{*}$ are the optimal power allocation values obtained from Theorem 5, $a_{5}=\frac{1}{K}\left(\theta_{b, p, \text { null }}+\theta_{m, \text { null }}\right)$, and $a_{6}=\frac{1}{K} \theta_{b, p, \text { null }}$, where

$$
\begin{aligned}
& \theta_{m, \text { null }}=\operatorname{tr}\left(\widehat{\mathbf{R}}_{0 m, \text { null }}^{0}\right) \\
& +\operatorname{tr}\left(\widehat{\mathbf{R}}_{0 m, \text { null }}^{0}\right)^{-1} \operatorname{tr}\left(\left(\mathbf{R}_{0 m, \text { null }}^{0}-\widehat{\mathbf{R}}_{0 m, \text { null }}^{0}\right) \widehat{\mathbf{R}}_{0 m, \text { null }}^{0}\right)
\end{aligned}
$$

and

$$
\begin{aligned}
\theta_{b, p, \text { null }}=\sum_{l=0}^{L} \sum_{k=1, k \neq m}^{K} \operatorname{tr} & \left(\widehat{\mathbf{R}}_{l k}^{l}\right)^{-1} \operatorname{tr}\left(\mathbf{R}_{0 m}^{l} \widehat{\mathbf{R}}_{l k}^{l}\right) \\
& +\sum_{l=1}^{L} \operatorname{tr}\left(\widehat{\mathbf{R}}_{l m, \text { null }}^{l}\right)^{-1} \Lambda_{0 m}^{l} .
\end{aligned}
$$

Furthermore, define $a_{7}=a_{3} a_{6}-a_{4} a_{5}, b_{7}=a_{6} b_{3}+a_{3}-$ $a_{5} b_{4}-a_{4}, c_{7}=a_{6}+b_{3}-a_{5}-b_{4}$, two fixed point equations for $\gamma$

$$
\begin{gathered}
\gamma=\min \left[\frac{-b_{7}+\sqrt{b_{7}^{2}-4 a_{7} c_{7}}}{2 a_{7}}, \frac{-b_{7}-\sqrt{b_{7}^{2}-4 a_{7} c_{7}}}{2 a_{7}}\right] \\
\gamma=\max \left[\frac{-b_{7}+\sqrt{b_{7}^{2}-4 a_{7} c_{7}}}{2 a_{7}}, \frac{-b_{7}-\sqrt{b_{7}^{2}-4 a_{7} c_{7}}}{2 a_{7}}\right],
\end{gathered}
$$

${ }^{3}$ When $a_{1}-a_{2}=0$, if $b_{1}-b_{2}>0$, then secure transmission can be achieved for any $p$; otherwise, secure transmission cannot be achieved regardless of the value of $p$. 


$$
p^{*}=\left\{\begin{array}{lll}
1, & \text { if } & p_{1} \notin[0,1], p_{2} \notin[0,1] \\
\arg \max \left\{R_{\text {sec, asy }}(1), R_{\text {sec, asy }}\left(p_{1}\right)\right\}, & \text { if } & p_{1} \in[0,1], p_{2} \notin[0,1] \\
\arg \max \left\{R_{\text {sec, asy }}(1), R_{\text {sec, asy }}\left(p_{1}\right)\right\}, & \text { if } & p_{1} \notin[0,1], p_{2} \in[0,1] \\
\arg \max \left\{R_{\text {sec, asy }}(1), R_{\text {sec, asy }}\left(p_{1}\right), R_{\text {sec, asy }}\left(p_{2}\right)\right\}, & \text { if } & p_{1} \in[0,1], p_{2} \in[0,1]
\end{array}\right.
$$

and $\Delta=b_{7}^{2}-4 a_{7} c_{7}$. We note that (66) and (67) are two fixed point equations since $a_{3}, b_{3}, a_{4}$, and $b_{4}$ are functions of $\gamma$. Denote the solution of (66) and (67) by $\gamma_{\mathrm{t}, 1}$ and $\gamma_{\mathrm{t}, 2}$, respectively, and define

$$
\beta(\gamma)=\left\{\begin{array}{l}
1, \text { if }\left(\Delta<0 \text { and } a_{7}<0\right) \text { or } \\
\left(\Delta>0, a_{7}>0 \text { and } \gamma_{\mathrm{t}, 1}<\gamma<\gamma_{\mathrm{t}, 2}\right) \text { or } \\
\left(\Delta>0, a_{7}<0 \text { and }\left(\gamma<\gamma_{\mathrm{t}, 1} \gamma>\text { oror } \gamma_{\mathrm{t}, 2}\right)\right) \\
0, \text { otherwise }
\end{array}\right.
$$

Then, we have the following theorem.

Theorem 7: For $N_{t} \rightarrow \infty$ and $N_{e}=1$, if $\beta(\gamma)=0$, the asymptotic secrecy rate of the MF-AN design is higher than that of the NS design; if $\beta(\gamma)=1$, the opposite is true.

Proof: Please refer to Appendix F.

\section{B. Single-Cell Single-User Case}

To provide more insights into the impact of the pilot contamination attack on secure communication in massive MIMO systems, we simplify the system model to the singlecell single-user case in this subsection. The transmitter (Alice) sends the desired signal to the receiver (Bob), and an eavesdropper (Eve) is present to overhear the signal, i.e., we have $L=0, K=1$, and $N_{e}=1$ [24-26]. We investigate again under which condition a positive secrecy rate is achievable. With the simplified model, in the following theorem, we obtain a more intuitive condition than the one in Theorem 6.

Theorem 8: For a single-cell single-user single-antenna eavesdropper system ( $\left.L=0, K=1, N_{e}=1\right)$, to achieve $R_{\mathrm{sec}, \text { asy }}>0$ in (24), the following condition must be satisfied:

$$
(p-1) \gamma \eta_{1}<\eta_{2},
$$

where

$$
\begin{aligned}
& \eta_{1}=\left[\left(\operatorname{tr}^{2}\left(\widehat{\mathbf{R}}_{01}^{0}\right)+\operatorname{tr}\left(\left(\mathbf{R}_{01}^{0}-\widehat{\mathbf{R}}_{01}^{0}\right) \widehat{\mathbf{R}}_{01}^{0}\right)\right)\right. \\
& \quad \times\left(\operatorname{tr}\left(\mathbf{R}_{E, T}^{0}\right) \operatorname{tr}\left(\widehat{\mathbf{R}}_{01}^{0}\right)-\Lambda\right) \\
& \left.-\left(\operatorname{tr}\left(\left(\mathbf{R}_{01}^{0}-\widehat{\mathbf{R}}_{01}^{0}\right) \widehat{\mathbf{R}}_{01}^{0}\right)+\operatorname{tr}\left(\widehat{\mathbf{R}}_{01}^{0}\right) \operatorname{tr}\left(\mathbf{R}_{01}^{0}-\widehat{\mathbf{R}}_{01}^{0}\right)\right) \Lambda\right]
\end{aligned}
$$

$$
\begin{gathered}
\eta_{2}= \\
{\left[\left(\operatorname{tr}^{2}\left(\widehat{\mathbf{R}}_{01}^{0}\right)+\operatorname{tr}\left(\left(\mathbf{R}_{01}^{0}-\widehat{\mathbf{R}}_{01}^{0}\right) \widehat{\mathbf{R}}_{01}^{0}\right)\right)-\Lambda\right] \operatorname{tr}\left(\widehat{\mathbf{R}}_{01}^{0}\right)} \\
\Lambda=\tau^{2} P_{01}^{2} \operatorname{tr}\left(\boldsymbol{\Omega}^{H} \mathbf{R}_{E, T}^{0} \boldsymbol{\Omega} \mathbf{R}_{01}^{0}\right)+\tau^{2} P_{01} P_{E}\left|\operatorname{tr}\left(\boldsymbol{\Omega} \mathbf{R}_{E, T}^{0}\right)\right|^{2} \\
+\tau P_{01} N_{0} \operatorname{tr}\left(\boldsymbol{\Omega}^{H} \mathbf{R}_{E, T}^{0} \boldsymbol{\Omega}\right)
\end{gathered}
$$

$$
\boldsymbol{\Omega}=\mathbf{R}_{01}^{0}\left(N_{0} \mathbf{I}_{N_{t}}+\tau\left(P_{01} \mathbf{R}_{01}^{0}+P_{E} \mathbf{R}_{E, T}^{0}\right)\right)^{-1} .
$$

Proof: Please refer to Appendix G.

Remark 5: From (69), we obtain that coefficients $\eta_{1}$ and $\eta_{2}$ determine whether secrecy can be achieved. In the high SNR regime when $\gamma \rightarrow \infty$, if $\eta_{1}>0$, (69) holds for arbitrary $p<1$ and secure communication can be achieved; if $\eta_{1}<0$, (69) holds only for $p>1$. However, $p$ denotes the fraction of power allocated to the transmit signal and satisfies $0<p \leq$ 1. This means that if $\eta_{1}<0$, (69) can not hold and secure communication can not be achieved.

In the following, we provide a concrete example for when $\eta_{1}<0$ may occur.

Theorem 9: Consider i.i.d. fading, i.e., $\mathbf{R}_{01}^{0}=\beta_{01} \mathbf{I}_{N_{t}}$ and $\mathbf{R}_{E, T}^{0}=\beta_{E} \mathbf{I}_{N_{t}}$, where $\beta_{01}$ and $\beta_{E}$ denote the path-losses for the desired user and the eavesdropper, respectively $[43,44]$. Then, $\eta_{1} \gtrless 0$ is equivalent to $P_{01} \beta_{01} \gtrless P_{E} \beta_{E}$.

Proof: Please refer to Appendix $\mathrm{H}$.

Theorems 8 and 9 imply that if the active eavesdropper increases the power of the pilot contamination attack, the classical MF-AN design [40] may not be able to achieve secure communication. Hence, a new design is needed to facilitate secure communication in massive MIMO systems.

Remark 6: If $\eta_{1}=0$ and $\eta_{2}>0$, then (69) is always satisfied. Hence, secure communication is achievable regardless of the pilot contamination power $P_{E}$ and SNR $\gamma$. The question is how $\eta_{1}=0$ and $\eta_{2}>0$ can be achieved. Clearly, in a conventional massive MIMO setting with i.i.d. channels, as in [34], this condition is satisfied with low probability, because for small-dimensional MIMO channels, both $\mathbf{R}_{01}^{0}$ and $\mathbf{R}_{E, T}^{0}$ in (70) are full rank positive definite matrices, such that $\eta_{1}=0$ in (70) is difficulty to achieve. However, a unique feature of massive MIMO channels is that the transmit correlation matrices of the channels are low rank $[3,4,6-8$, $10,38]$. Therefore, for massive MIMO systems, it is possible to perform joint uplink and downlink processing as in Section III-B to project the channels of the users and the eavesdropper into the null space of $\mathbf{R}_{E, T}^{0}$. For this NS design, the condition in (69) still holds after replacing $\mathbf{R}_{01}^{0}$ and $\mathbf{R}_{E, T}^{0}$ in (70) and (71) with $\mathbf{R}_{01, \text { null }}^{0}$ and $\mathbf{0}$, respectively. In this case, we obtain $\eta_{1}=0$ and $\eta_{2}>0$. In fact, this is the intuition behind the NS design proposed in this paper. Unlike the conventional NS design for the perfect CSI case [24], the proposed NS design transmits along the statistical eigen-direction of the channel. Moreover, since the pilot contamination attack affects both the uplink channel estimation and the downlink data transmission, the proposed NS design requires a joint uplink and downlink processing to completely eliminate the impact of the pilot contamination attack, see Section III-B for details. 


\section{NumERICAL RESULTS}

In this section, we provide numerical results to evaluate the secrecy performance of the considered massive MIMO system with an active eavesdropper. We consider a system where a uniform linear array with $N_{t}=128$ is employed at the BS, the antenna spacing is half a wavelength, and the angle of arrival (AoA) interval is $\mathcal{A}=[-\pi, \pi]$. We use the truncated Laplacian distribution to model the channel power angle spectrum as [51]

$$
p(\theta)=\frac{1}{\sqrt{2} \sigma\left(1-e^{-\sqrt{2} \pi / \sigma}\right)} e^{\frac{-\sqrt{2}\|\theta-\bar{\theta}\|}{\sigma}},
$$

where $\sigma$ and $\bar{\theta}$ denote the angular spread (AS) and the mean AoA of the channel, respectively. We assume that the AS $\sigma$ in (74) is identical for the channels of all users and the eavesdropper and we set $\sigma=\pi / 2$. The channel transmit correlation matrices of all users, $\mathbf{R}_{l k}^{l}$, and the eavesdropper, $\mathbf{R}_{E, T}^{l}$, are generated based on [51, Eq. (3.14)]. For the channel between the user and the BS in its own cell and the channel between the user and the BSs in the other cells, we impose a channel power normalization to make the trace of the channel transmit correlation matrices equal to $N_{t}$ and $\rho N_{t}$, respectively, and set $\rho=0.1$. The receive correlation matrices of the eavesdropper, $\mathbf{R}_{E, R}^{l}$, are generated using the exponential correlation model $\left\{\mathbf{R}_{E, R}^{l}\right\}_{i, j}=\varphi^{|i-j|}, \varphi \in(0,1)$, where $\varphi$ is generated at random. We note that for any precoder $\mathbf{P}_{e}$ satisfying (13), the resulting $r_{E, R}^{0}$ in (3) and (5) is the same. In the simulations, we set $\mathbf{p}_{s}=\sqrt{\frac{1}{N_{e}}} \mathbf{u}_{e}, s=1,2, \cdots, N_{e}$. For the channel between the eavesdropper and the 0th BS and the channel between the eavesdropper and the other BSs, we impose a channel power normalization to make the trace of the channel receive correlation matrices equal to $N_{e}$ and $\rho N_{e}$, respectively. The asymptotic secrecy rate is computed based on Theorem 2 and the exact secrecy rate is obtained by Monte Carlo simulation. We set $L=3, K=5, P_{l k}=1$, $k=1,2, \cdots, K, l=0,1, \cdots, L, \tau=10$, and $N_{0}=1$. The mean channel AoAs, $\bar{\theta}$, of all users and the eavesdropper in (74) are generated at random and the channel AoAs, $\theta$, of all users and the eavesdropper are distributed within the angle interval $[-\pi, \pi]$ according to (74).

Figure 4 shows the secrecy rate performance versus (vs.) SNR $\gamma$ for the MF-AN design, $P_{E}=1$, different $p$, and different $N_{e}$. We observe from Figure 4 that the asymptotic secrecy rate in Theorem 2 provides a good estimate for the exact secrecy rate. Also, we observe from Figure 4 that in the low SNR regime, allocating more power (larger $p$ ) to the information-carrying transmit signal leads to a higher secrecy rate. However, as the SNR increases, the secrecy rate drops significantly if the transmit signal power is high and the AN power is small. For example, for $\mathrm{SNR}=2 \mathrm{~dB}$ and $N_{e}=1$, we find from Theorem 6 that $p<0.15$ is a necessary condition to guarantee reliable communication. Thus, for $p=0.16$, a positive secrecy rate cannot be achieved, as confirmed by Figure 4. In addition, we observe from Figure 4 that for the MF-AN design, increasing $N_{e}$ results in a secrecy rate degradation.

Figure 5 shows the secrecy rate performance vs. $p$ for the MF-AN design, $N_{e}=1, P_{E}=1$, and different SNRs. For

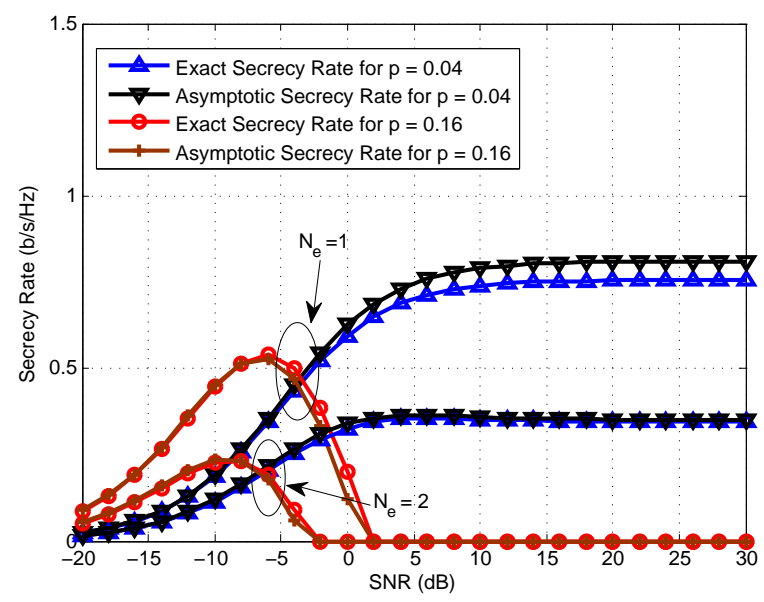

Fig. 4: Secrecy rate vs. SNR for $P_{E}=1$, different $p$, and different $N_{e}$.

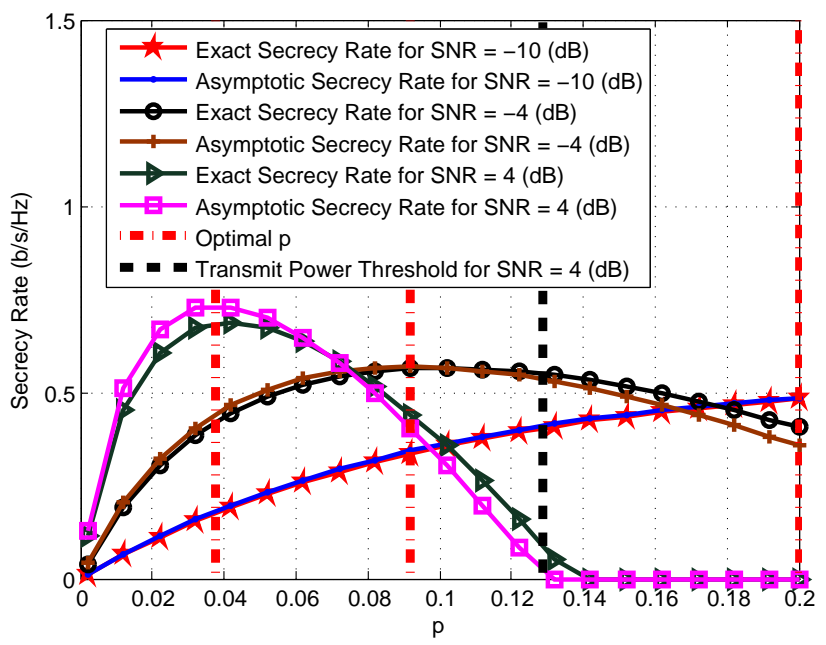

Fig. 5: Secrecy rate vs. $p$ for $N_{e}=1, P_{E}=1$, and different SNRs.

$K=5$, we know from Section II-B that $0 \leq p \leq 0.2$. Figure 5 confirms that the asymptotic secrecy rate in Theorem 2 provides a good estimate for the exact secrecy rate. Also, we observe from Figure 5 that the maximum of the exact secrecy rate is achieved for the optimal power allocation solution provided in Theorem 5. Furthermore, for $\mathrm{SNR}=4$ $\mathrm{dB}$, a positive secrecy rate cannot be achieved when $p$ is larger than the analytical transmit power threshold provided in Theorem 6. As the SNR increases, more power has to be used for generating $\mathrm{AN}$, in order to achieve reliable secrecy transmission.

Figure 6 shows the exact secrecy rate performance vs. SNR $\gamma$ for $N_{e}=1, P_{E}=1$, and different system designs. For the MF-AN design, we adopted the optimal power allocation $p$ and $q$ based on Theorem 5. For the NS design, when an eigenvalue of $\mathbf{R}_{E, T}^{l}$ is less than $10^{-3}$, we treated it as being equal to zero. Based on this, we obtained $T_{l}$ and $\mathbf{V}_{E}^{l}$ according to Section III-B. For the unified design, we obtained the optimal $\alpha$ and $\beta$ by performing a one-dimensional numerical 


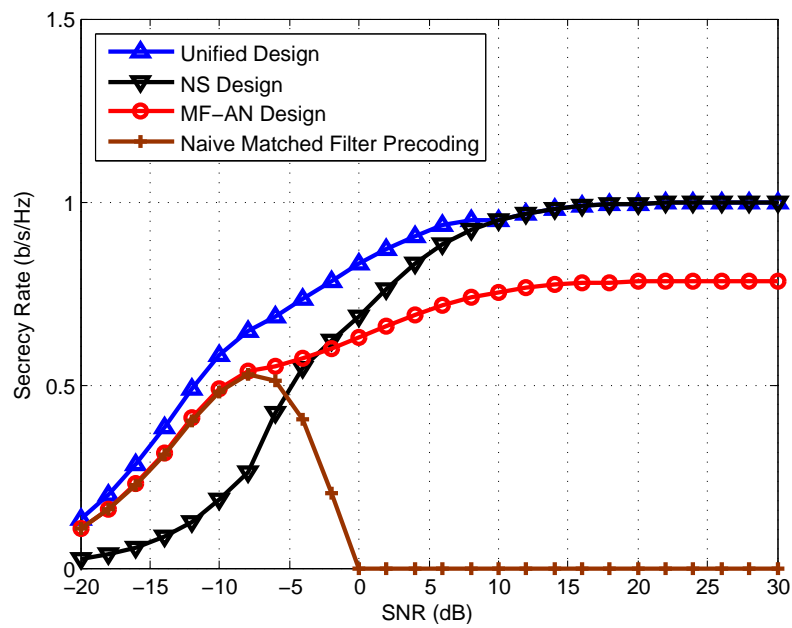

Fig. 6: Exact secrecy rate vs. the SNR for $N_{e}=1, P_{E}=1$, and different precoding designs.

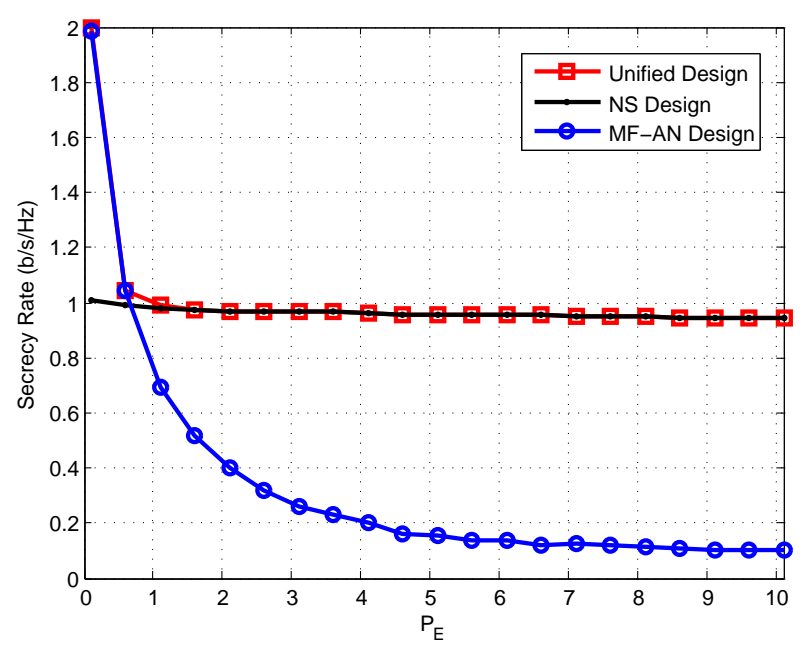

Fig. 7: Exact secrecy rate vs. $P_{E}$ for $N_{e}=1, \gamma=10 \mathrm{~dB}$, and different precoding designs.

search. For comparison, we also show results for a naive MF precoding scheme, where $p=1 / K$ and AN is not generated. From Figure 6, we make the following observations: 1) The unified design achieves the best performance for all considered SNR values. 2) Because of the pilot contamination attack, even though the transmitter is equipped with a large number of antennas, naive MF precoding cannot achieve a positive secrecy rate for moderate-to-high SNRs.

Figure 7 shows the exact secrecy rate performance vs. $P_{E}$ for $N_{e}=1, \gamma=10 \mathrm{~dB}$, and different system designs. We observe from Figure 7 that the unified design performs best for all considered $P_{E}$ values. For a weak pilot contamination attack, the MF-AN design performs better than the NS design. However, when the eavesdropper increases its pilot power, this leads to a serious secrecy rate loss for the MF-AN design, but has barely any impact on the NS design. This is because the NS design can eliminate the impact of the pilot contamination caused by the active eavesdropper as suggested by (48) where

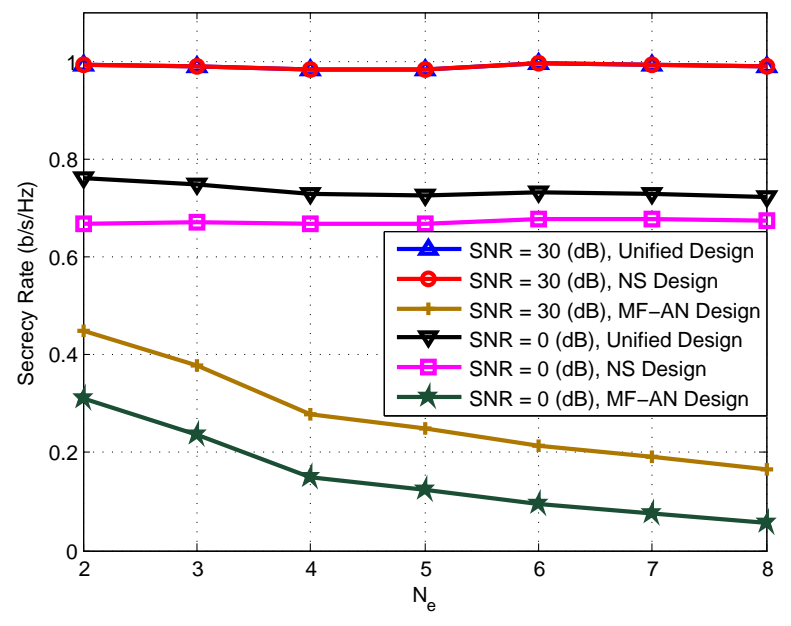

Fig. 8: Exact secrecy rate vs. $N_{e}$ for $P_{E}=1$, different system designs, and different SNRs.

the term $P_{E} \mathbf{R}_{E, T}^{0}$ disappears in the covariance matrix of the estimated channel. When $P_{E}=0.1$ and $P_{E}=1$, Theorem 7 indicates that, for $\gamma=10 \mathrm{~dB}$, the MF-AN design and the NS design perform better, respectively, which is confirmed by Figure 7.

Figure 8 shows the exact secrecy rate performance vs. $N_{e}$ for $P_{E}=1$, different system designs, and different SNRs. We observe from Figure 8 that the unified design performs best for the considered SNR values. Also, the secrecy rate of the unified design barely decreases with increasing $N_{e}$. This confirms that the unified design is an effective approach for combating the pilot contamination attack. In contrast, the secrecy rate performance of the MF-AN design degrades significantly with increasing $N_{e}$.

\section{ACKNOWLEDGMENT}

The authors would like to thank Prof. Xiqi Gao, Prof. C. K. Wen, and Mr. Shahram Zarei for the helpful discussion throughout the paper, Prof. Shi Jin for pointing out [44], and Prof. Jiaheng Wang for the helpful discussion on Theorem 1. The authors would also like to thank the editor, Prof. Yingbin Liang, and anonymous reviewers for helpful comments and suggestions that greatly improve the quality of the paper.

\section{Conclusions}

In this paper, we have studied the transmit signal design for multi-cell multi-user massive MIMO systems in the presence of a multi-antenna active eavesdropper. For the MF-AN design, we obtained an asymptotic achievable secrecy rate expression for the pilot contamination attack when the number of transmit antennas tends to infinity. Moreover, we proved that the impact of the active eavesdropper can be completely eliminated when the transmit correlation matrices of the users and the eavesdropper are orthogonal. This analytical insight motivated the development of transmit signal designs that are robust against the pilot contamination attack. Also, for the MF-AN design and a single-antenna eavesdropper, we derived closed-form 
expressions for the optimal power allocation policy for the information-carrying transmit signal and the $\mathrm{AN}$ as well as the minimum transmit signal power required to ensure secure transmission. In addition, for the single-antenna eavesdropper case, a decision threshold for determining whether the MF-AN design or the NS design is preferable was provided. Monte Carlo simulation results showed that the derived analytical results are accurate and confirmed the effectiveness of the proposed transmission schemes for combating the pilot contamination attack.

\section{APPENDIX A}

\section{ProOF OF THEOREM 1}

First, recalling the definition of $\mathbf{W}_{e}$, we have

$$
\mathbf{W}_{e} \mathbf{W}_{e}^{H}=\tau \mathbf{1}_{N_{e}} \mathbf{1}_{N_{e}}^{T} .
$$

Then, the constraint in (II-A) reduces to

$$
\operatorname{tr}\left(\mathbf{P}_{e} \mathbf{1}_{N_{e}} \mathbf{1}_{N_{e}}^{T} \mathbf{P}_{e}^{H}\right)=\operatorname{tr}\left(\left(\sum_{r=1}^{N_{r}} \mathbf{p}_{r}\right)\left(\sum_{r=1}^{N_{r}} \mathbf{p}_{r}\right)^{H}\right) \leq N_{e}
$$

Also, (12) can be re-written as

$$
\begin{aligned}
\sum_{r=1}^{N_{r}} \sum_{s=1}^{N_{r}}\left\{\mathbf{P}_{e}^{H} \mathbf{R}_{E, R}^{0} \mathbf{P}_{e}\right\}_{r s} & =\sum_{r=1}^{N_{r}} \sum_{s=1}^{N_{r}} \mathbf{e}_{r}^{H} \mathbf{P}_{e}^{H} \mathbf{R}_{E, R}^{0} \mathbf{P}_{e} \mathbf{e}_{s} \\
& =\sum_{r=1}^{N_{r}} \sum_{s=1}^{N_{r}} \mathbf{p}_{r}^{H} \mathbf{R}_{E, R}^{0} \mathbf{p}_{s} \\
& =\left(\sum_{r=1}^{N_{r}} \mathbf{p}_{r}^{H}\right) \mathbf{R}_{E, R}^{0}\left(\sum_{s=1}^{N_{r}} \mathbf{p}_{s}\right)
\end{aligned}
$$

Define $\mathbf{p}=\sum_{r=1}^{N_{e}} \mathbf{p}_{r}$. Considering (76), (77), the optimization problem in (12) is equivalent to

$$
\begin{gathered}
\max _{\mathbf{p}} \mathbf{p}^{H} \mathbf{R}_{E, R}^{0} \mathbf{p} \\
\text { s.t. } \operatorname{tr}\left(\mathbf{p} \mathbf{p}^{H}\right) \leq N_{e} .
\end{gathered}
$$

It is easy to show that the optimal solution of (78) is $\mathbf{p}=\sqrt{N_{e}} \mathbf{u}_{e}$, where $\mathbf{u}_{e}$ is the eigenvector corresponding to the largest eigenvalue of $\mathbf{R}_{E, R}^{0}$. This completes the proof.

\section{APPENDIX B \\ PROOF OF THEOREM 2}

First, we calculate $\operatorname{SINR}_{0 m}$ in (20) for $N_{t} \rightarrow \infty$. For the numerator of (20), based on [52, Corollary 1], we have

$$
\begin{aligned}
& \frac{1}{N_{t}}\left[\left|\left(\mathbf{h}_{0 m}^{0}\right)^{H} \mathbf{w}_{0 m}\right|^{2}\right] \\
& \stackrel{N_{t} \rightarrow \infty}{\rightarrow} \frac{1}{N_{t}}\left|\widehat{\mathbf{h}}_{0 m}^{0}\right|^{2}+\frac{1}{N_{t}}\left[\frac{\left(\widehat{\mathbf{h}}_{0 m}^{0}\right)^{H}\left(\mathbf{R}_{0 m}^{0}-\widehat{\mathbf{R}}_{0 m}^{0}\right) \widehat{\mathbf{h}}_{0 m}^{0}}{\left\|\widehat{\mathbf{h}}_{0 m}^{0}\right\|^{2}}\right] \\
& \stackrel{N_{t} \rightarrow \infty}{\rightarrow} \frac{1}{N_{t}} \operatorname{tr}\left(\widehat{\mathbf{R}}_{0 m}^{0}\right)+\frac{1}{N_{t}} \frac{\operatorname{tr}\left(\left(\mathbf{R}_{0 m}^{0}-\widehat{\mathbf{R}}_{0 m}^{0}\right) \widehat{\mathbf{R}}_{0 m}^{0}\right)}{\operatorname{tr}\left(\widehat{\mathbf{R}}_{0 m}^{0}\right)} .
\end{aligned}
$$

For the denominator of (20), we have

$$
\begin{aligned}
\frac{1}{N_{t}}\left|\left(\mathbf{h}_{0 m}^{0}\right)^{H} \mathbf{w}_{0 k}\right|^{2} & =\frac{\left(\mathbf{h}_{0 m}^{0}\right)^{H} \widehat{\mathbf{h}}_{0 k}^{0}\left(\widehat{\mathbf{h}}_{0 k}^{0}\right)^{H}\left(\mathbf{h}_{0 m}^{0}\right)}{\left(\widehat{\mathbf{h}}_{0 k}^{0}\right)^{H} \widehat{\mathbf{h}}_{0 k}^{0}} \\
& \stackrel{N_{t} \rightarrow \infty}{\rightarrow} \frac{\left(\widehat{\mathbf{h}}_{0 k}^{0}\right)^{H} \mathbf{R}_{0 m}^{0} \widehat{\mathbf{h}}_{0 k}^{0}}{\left(\widehat{\mathbf{h}}_{0 k}^{0}\right)^{H} \widehat{\mathbf{h}}_{0 k}^{0}} \\
& \stackrel{N_{t} \rightarrow \infty}{\rightarrow} \frac{\operatorname{tr}\left(\mathbf{R}_{0 m}^{0} \widehat{\mathbf{R}}_{0 k}^{0}\right)}{\operatorname{tr}\left(\widehat{\mathbf{R}}_{0 k}^{0}\right)} .
\end{aligned}
$$

Also, performing some simplifications, we obtain

$$
\begin{aligned}
\frac{1}{N_{t}} & {\left[\left\|\left(\mathbf{h}_{0 m}^{0}\right)^{H} \mathbf{U}_{\text {null }, 0}\right\|^{2}\right] \stackrel{N_{t} \rightarrow \infty}{\rightarrow} \frac{1}{N_{t}} \mathbf{R}_{0 m}^{0} } \\
- & \frac{1}{N_{t}} \sum_{k=1, k \neq m}^{K} \operatorname{tr}\left(\widehat{\mathbf{R}}_{0 k}^{0}\right)^{-1}\left(\mathbf{h}_{0 m}^{0}\right)^{H}\left(\widehat{\mathbf{h}}_{0 k}^{0}\right)\left(\widehat{\mathbf{h}}_{0 k}^{0}\right)^{H} \mathbf{h}_{0 m}^{0} \\
& -\frac{1}{N_{t}} \operatorname{tr}\left(\widehat{\mathbf{R}}_{0 m}^{0}\right)^{-1}\left(\mathbf{h}_{0 m}^{0}\right)^{H}\left(\widehat{\mathbf{h}}_{0 m}^{0}\right)\left(\widehat{\mathbf{h}}_{0 m}^{0}\right)^{H} \mathbf{h}_{0 m}^{0} .
\end{aligned}
$$

For $m \neq k, \mathbf{h}_{0 m}^{0}$ is independent of $\widehat{\mathbf{h}}_{0 k}^{0}$. Hence, the asymptotic expression for $\frac{1}{N_{t}}\left(\mathbf{h}_{0 m}^{0}\right)^{H} \widehat{\mathbf{h}}_{0 k}^{0}\left(\widehat{\mathbf{h}}_{0 k}^{0}\right)^{H} \mathbf{h}_{0 m}^{0}$ is given by

$$
\frac{1}{N_{t}}\left(\mathbf{h}_{0 m}^{0}\right)^{H} \widehat{\mathbf{h}}_{0 k}^{0}\left(\widehat{\mathbf{h}}_{0 k}^{0}\right)^{H} \mathbf{h}_{0 m}^{0} \stackrel{N_{t} \rightarrow \infty}{\rightarrow} \frac{1}{N_{t}} \operatorname{tr}\left(\mathbf{R}_{0 m}^{0} \widehat{\mathbf{R}}_{0 k}^{0}\right) .
$$

For $m=k$, based on (3), we have

$$
\begin{aligned}
\widehat{\mathbf{h}}_{0 m}^{0}=\sqrt{P_{0 m}} & \mathbf{C}_{0 m}^{0} \mathbf{h}_{0 m}^{0}+\mathbf{C}_{0 m}^{0} \sum_{l=1}^{L} \sqrt{P_{l m}} \tau \mathbf{h}_{l m}^{0} \\
+ & \mathbf{C}_{0 m}^{0} \sqrt{\frac{P_{E}}{N_{e}}} \tau \sum_{r=1}^{N_{e}} \mathbf{h}_{E, r}^{0}+\mathbf{C}_{0 m}^{0} \boldsymbol{\Omega}_{m} \mathbf{n},
\end{aligned}
$$

where $\mathbf{C}_{0 m}^{0}$ is defined in (31) and $\boldsymbol{\Omega}_{m}=\left(\boldsymbol{\omega}_{m} \otimes \mathbf{I}_{N_{t}}\right)^{H}$.

Based on (83), we obtain

$$
\begin{gathered}
\left(\mathbf{h}_{0 m}^{0}\right)^{H} \widehat{\mathbf{h}}_{0 m}^{0}\left(\widehat{\mathbf{h}}_{0 m}^{0}\right)^{H} \mathbf{h}_{0 m}^{0} \\
=\left(\mathbf{h}_{0 m}^{0}\right)^{H} \mathbf{C}_{0 m}^{0} \boldsymbol{\Omega}_{m} \mathbf{n}\left(\widehat{\mathbf{h}}_{0 m}^{0}\right)^{H} \mathbf{h}_{0 m}^{0}+\tau^{2}\left(\mathbf{h}_{0 m}^{0}\right)^{H} \mathbf{C}_{0 m}^{0} \\
\times \sum_{t=0}^{L} \sum_{s=0}^{L} \sqrt{P_{t m}} \sqrt{P_{s m}} \mathbf{h}_{t m}^{0}\left(\mathbf{h}_{s m}^{0}\right)^{H}\left(\mathbf{C}_{0 m}^{0}\right)^{H} \mathbf{h}_{0 m}^{0}+\tau^{2} \sqrt{\frac{P_{E}}{N_{e}}} \\
\times\left(\mathbf{h}_{0 m}^{0}\right)^{H} \mathbf{C}_{0 m}^{0} \sum_{t=0}^{L} \sqrt{P_{t m}} \mathbf{h}_{t m}^{0} \sum_{r=1}^{N_{e}}\left(\mathbf{h}_{E, r}^{0}\right)^{H}\left(\mathbf{C}_{0 m}^{0}\right)^{H} \mathbf{h}_{0 m}^{0} \\
+\tau\left(\mathbf{h}_{0 m}^{0}\right)^{H} \mathbf{C}_{0 m}^{0} \sum_{t=0}^{L} \sqrt{P_{t m}} \mathbf{h}_{t m}^{0} \mathbf{h}^{H} \boldsymbol{\Omega}_{m}^{H}\left(\mathbf{C}_{0 m}^{0}\right)^{H} \mathbf{h}_{0 m}^{0} \\
\quad+\tau \sqrt{P_{E}}\left(\mathbf{h}_{0 m}^{0}\right)^{H} \mathbf{C}_{0 m}^{0} \sum_{r=1}^{N_{e}} \mathbf{h}_{E, r}^{0}\left(\widehat{\mathbf{h}}_{0 m}^{0}\right)^{H} \mathbf{h}_{0 m}^{0} .
\end{gathered}
$$

When $N_{t} \rightarrow \infty$, based on (84) and [52, Corollary 1], we 
have

$$
\begin{aligned}
& \frac{1}{N_{t}}\left(\mathbf{h}_{0 m}^{0}\right)^{H} \widehat{\mathbf{h}}_{0 m}^{0}\left(\widehat{\mathbf{h}}_{0 m}^{0}\right)^{H} \mathbf{h}_{0 m}^{0} \\
& \stackrel{N_{t} \rightarrow \infty}{\rightarrow} \frac{1}{N_{t}} \tau^{2}\left(\mathbf{h}_{0 m}^{0}\right)^{H} \mathbf{C}_{0 m}^{0} \sum_{t=0}^{L} P_{t m} \mathbf{h}_{t m}^{0}\left(\mathbf{h}_{t m}^{0}\right)^{H}\left(\mathbf{C}_{0 m}^{0}\right)^{H} \mathbf{h}_{0 m}^{0} \\
& +\frac{1}{N_{t}} \tau^{2} \frac{P_{E}}{N_{e}}\left(\mathbf{h}_{0 m}^{0}\right)^{H} \mathbf{C}_{0 m}^{0} \sum_{r=1}^{N_{e}} \mathbf{h}_{E, r}^{0} \sum_{r=1}^{N_{e}}\left(\mathbf{h}_{E, r}^{0}\right)^{H}\left(\mathbf{C}_{0 m}^{0}\right)^{H} \mathbf{h}_{0 m}^{0} \\
& +\frac{1}{N_{t}}\left(\mathbf{h}_{0 m}^{0}\right)^{H} \mathbf{C}_{0 m}^{0} \boldsymbol{\Omega}_{m} \mathbf{n}\left(\mathbf{C}_{0 m}^{0} \boldsymbol{\Omega}_{m} \mathbf{n}\right)^{H} \mathbf{h}_{0 m}^{0} \\
& \stackrel{N_{t} \rightarrow \infty}{\rightarrow} \frac{1}{N_{t}} \tau^{2} P_{0 m}\left(\mathbf{h}_{0 m}^{0}\right)^{H} \mathbf{C}_{0 m}^{0} \mathbf{h}_{0 m}^{0}\left(\mathbf{h}_{0 m}^{0}\right)^{H}\left(\mathbf{C}_{0 m}^{0}\right)^{H} \mathbf{h}_{0 m}^{0} \\
& +\frac{1}{N_{t}} \tau^{2}\left(\mathbf{h}_{0 m}^{0}\right)^{H} \mathbf{C}_{0 m}^{0} \sum_{t=1}^{L} P_{t m} \mathbf{h}_{t m}^{0}\left(\mathbf{h}_{t m}^{0}\right)^{H}\left(\mathbf{C}_{0 m}^{0}\right)^{H} \mathbf{h}_{0 m}^{0} \\
& +\frac{1}{N_{t}} \tau^{2} \frac{P_{E}}{N_{e}}\left(\mathbf{h}_{0 m}^{0}\right)^{H} \mathbf{C}_{0 m}^{0} \sum_{r=1}^{N_{e}} \mathbf{h}_{E, r}^{0} \sum_{r=1}^{N_{e}}\left(\mathbf{h}_{E, r}^{0}\right)^{H}\left(\mathbf{C}_{0 m}^{0}\right)^{H} \mathbf{h}_{0 m}^{0} \\
& +\frac{1}{N_{t}}\left(\mathbf{h}_{0 m}^{0}\right)^{H} \mathbf{C}_{0 m}^{0} \boldsymbol{\Omega}_{m} \mathbf{n}\left(\mathbf{C}_{0 m}^{0} \boldsymbol{\Omega}_{m} \mathbf{n}\right)^{H} \mathbf{h}_{0 m}^{0}{ }^{N_{t} \rightarrow \infty} \rightarrow \frac{1}{N_{t}} \Lambda_{0 m}^{0} .
\end{aligned}
$$

Also, for $m \neq k, \mathbf{h}_{0 m}^{l}$ is independent of $\widehat{\mathbf{h}}_{l k}^{l}$, and we obtain in (21)

$$
\begin{aligned}
& \frac{1}{N_{t}}\left|\left(\mathbf{h}_{0 m}^{l}\right)^{H} \mathbf{w}_{l k}\right|^{2} \\
& =\frac{1}{N_{t}}\left(\mathbf{h}_{0 m}^{l}\right)^{H} \frac{\widehat{\mathbf{h}}_{l k}^{l}}{\left|\widehat{\mathbf{h}}_{l k}^{l}\right|} \frac{\left(\widehat{\mathbf{h}}_{l k}^{l}\right)^{H}}{\left|\widehat{\mathbf{h}}_{l k}^{l}\right|} \mathbf{h}_{0 m}^{l} \\
& \stackrel{N_{t \rightarrow \infty}}{\rightarrow} \frac{\left(\widehat{\mathbf{h}}_{l k}^{l}\right)^{H} \mathbf{R}_{0 m}^{l} \widehat{\mathbf{h}}_{l k}^{l}}{\left|\widehat{\mathbf{h}}_{l k}^{l}\right|^{2}} \stackrel{N_{t} \rightarrow \infty}{\rightarrow} \frac{\operatorname{tr}\left(\mathbf{R}_{0 m}^{l} \widehat{\mathbf{R}}_{l k}^{l}\right)}{\operatorname{tr}\left(\widehat{\mathbf{R}}_{l k}^{l}\right)} .
\end{aligned}
$$

For $m=k$, similar to (85), we have

$$
\begin{aligned}
\frac{1}{N_{t}}\left|\left(\mathbf{h}_{0 m}^{l}\right)^{H} \mathbf{w}_{l m}\right|^{2} & =\frac{1}{N_{t}} \frac{\left(\mathbf{h}_{0 m}^{l}\right)^{H} \widehat{\mathbf{h}}_{l m}^{l}\left(\widehat{\mathbf{h}}_{l m}^{l}\right)^{H} \mathbf{h}_{0 m}^{l}}{\left|\widehat{\mathbf{h}}_{l m}^{l}\right|^{2}} \\
& \stackrel{N_{t} \rightarrow \infty}{\rightarrow} \frac{1}{N_{t}} \frac{\Lambda_{0 m}^{l} \operatorname{tr}\left(\widehat{\mathbf{R}}_{l m}^{l}\right)}{}
\end{aligned}
$$

Next, we simplify

$$
\begin{aligned}
& \left(\mathbf{h}_{0 m}^{l}\right)^{H} \mathbf{U}_{\text {null }, l} \mathbf{U}_{\text {null }, l}^{H} \mathbf{h}_{0 m}^{l}= \\
& \operatorname{tr}\left(\mathbf{R}_{0 m}^{l}\right)-\sum_{k=1, k \neq m}^{K} \operatorname{tr}\left(\widehat{\mathbf{R}}_{l k}^{l}\right)^{-1}\left(\mathbf{h}_{0 m}^{l}\right)^{H}\left(\widehat{\mathbf{h}}_{l k}^{l}\right)\left(\widehat{\mathbf{h}}_{l k}^{l}\right)^{H} \mathbf{h}_{0 m}^{l} \\
& -\operatorname{tr}\left(\widehat{\mathbf{R}}_{l m}^{l}\right)^{-1}\left(\mathbf{h}_{0 m}^{l}\right)^{H}\left(\widehat{\mathbf{h}}_{l m}^{l}\right)\left(\widehat{\mathbf{h}}_{l m}^{l}\right)^{H} \mathbf{h}_{0 m}^{l} .
\end{aligned}
$$

Following a similar approach as was used to obtain (82) and (85), we obtain

$$
\begin{aligned}
& \frac{1}{N_{t}}\left(\mathbf{h}_{0 m}^{l}\right)^{H}\left(\widehat{\mathbf{h}}_{l k}^{l}\right)\left(\widehat{\mathbf{h}}_{l k}^{l}\right)^{H} \mathbf{h}_{0 m}^{l} \stackrel{N_{t} \rightarrow \infty}{\rightarrow} \operatorname{tr}\left(\mathbf{R}_{0 m}^{l} \widehat{\mathbf{R}}_{l k}^{l}\right) \\
& \frac{1}{N_{t}}\left(\mathbf{h}_{0 m}^{l}\right)^{H}\left(\widehat{\mathbf{h}}_{l m}^{l}\right)\left(\widehat{\mathbf{h}}_{l m}^{l}\right)^{H} \mathbf{h}_{0 m}^{l} \stackrel{N_{t} \rightarrow \infty}{\rightarrow} \Lambda_{0 m}^{l} .
\end{aligned}
$$

By substituting (79)-(90) into (20), we obtain the expression for $\mathrm{SINR}_{0 m \text {, asy }}$ in (25).

Next, we simplify (22). First, we have

$$
\begin{aligned}
\left(\mathbf{H}_{E}^{l}\right)^{H} \mathbf{U}_{\text {null }, l} \mathbf{U}_{\text {null }, l}^{H} \mathbf{H}_{E}^{l} \\
=\left(\mathbf{H}_{E}^{l}\right)^{H}\left(\mathbf{I}_{N_{t}}-\widehat{\mathbf{H}}_{l}^{l} \operatorname{diag}\left[\operatorname{tr}\left(\widehat{\mathbf{R}}_{l 1}^{l}\right)^{-1}, \operatorname{tr}\left(\widehat{\mathbf{R}}_{l 2}^{l}\right)^{-1}, \cdots,\right.\right. \\
\left.\left.\quad \operatorname{tr}\left(\widehat{\mathbf{R}}_{l K}^{l}\right)^{-1}\right]\left(\widehat{\mathbf{H}}_{l}^{l}\right)^{H}\right) \mathbf{H}_{E}^{l}=\left(\mathbf{H}_{E}^{l}\right)^{H} \mathbf{H}_{E}^{l}-\mathbf{Q}_{H}
\end{aligned}
$$

where

$$
\begin{aligned}
& \mathbf{Q}_{H}=\left(\mathbf{H}_{E}^{l}\right)^{H} \widehat{\mathbf{H}}_{l}^{l} \\
& \times \operatorname{diag}\left[\operatorname{tr}\left(\widehat{\mathbf{R}}_{l 1}^{l}\right)^{-1}, \operatorname{tr}\left(\widehat{\mathbf{R}}_{l 2}^{l}\right)^{-1}, \cdots, \operatorname{tr}\left(\widehat{\mathbf{R}}_{l K}^{l}\right)^{-1}\right]\left(\widehat{\mathbf{H}}_{l}^{l}\right)^{H} \mathbf{H}_{E}^{l} .
\end{aligned}
$$

For $\left(\mathbf{H}_{E}^{l}\right)^{H} \mathbf{H}_{E}^{l}$, we have

$$
\begin{aligned}
& \begin{array}{l}
\left\{\left(\mathbf{H}_{E}^{l}\right)^{H} \mathbf{H}_{E}^{l}\right\}_{i j}=\left(\mathbf{h}_{E, i}^{l}\right)^{H} \mathbf{h}_{E, j}^{l} \\
=\mathbf{e}_{i}^{H}\left(\mathbf{R}_{E, R}^{l}\right)^{1 / 2}\left(\mathbf{G}_{E}^{l}\right)^{H}\left(\mathbf{R}_{E, T}^{l}\right)^{1 / 2} \\
\quad \times\left(\mathbf{R}_{E, T}^{l}\right)^{1 / 2} \mathbf{G}_{E}^{l}\left(\mathbf{R}_{E, R}^{l}\right)^{1 / 2} \mathbf{e}_{j}
\end{array} \\
& \begin{aligned}
= & \operatorname{tr}\left(\mathbf{e}_{i}^{H}\left(\mathbf{R}_{E, R}^{l}\right)^{1 / 2}\left(\mathbf{G}_{E}^{l}\right)^{H}\left(\mathbf{R}_{E, T}^{l}\right)^{1 / 2}\right. \\
& \left.\times\left(\mathbf{R}_{E, T}^{l}\right)^{1 / 2} \mathbf{G}_{E}^{l}\left(\mathbf{R}_{E, R}^{l}\right)^{1 / 2} \mathbf{e}_{j}\right)
\end{aligned} \\
& \stackrel{N_{t} \rightarrow \infty}{\rightarrow} \operatorname{tr}\left(\left(\mathbf{R}_{E, R}^{l}\right)^{1 / 2} \mathbf{e}_{j} \mathbf{e}_{i}^{H}\left(\mathbf{R}_{E, R}^{l}\right)^{1 / 2}\right) \operatorname{tr}\left(\mathbf{R}_{E, T}^{l}\right) \\
& =\left\{\mathbf{R}_{E, R}^{l}\right\}_{i j} \operatorname{tr}\left(\mathbf{R}_{E, T}^{l}\right),
\end{aligned}
$$

where we used [53, Eq. (102)]. Then, performing some simplifications, we get

$$
\left\{\mathbf{Q}_{H}\right\}_{i j}=\sum_{k=1}^{K} \operatorname{tr}\left(\widehat{\mathbf{R}}_{l k}^{l}\right)^{-1}\left(\mathbf{h}_{E, i}^{l}\right)^{H}\left(\widehat{\mathbf{h}}_{l k}^{l}\right)\left(\widehat{\mathbf{h}}_{l k}^{l}\right)^{H} \mathbf{h}_{E, j}^{l} .
$$

For $m \neq k, \mathbf{h}_{E, i}^{l}$ and $\mathbf{h}_{E, j}^{l}$ are independent of $\widehat{\mathbf{h}}_{l k}^{l}$. Thus, we have

$$
\begin{gathered}
\left(\mathbf{h}_{E, i}^{l}\right)^{H} \widehat{\mathbf{h}}_{l k}^{l}\left(\widehat{\mathbf{h}}_{l k}^{l}\right)^{H} \mathbf{h}_{E, j}^{l}=\left(\widehat{\mathbf{h}}_{l k}^{l}\right)^{H} \mathbf{h}_{E, j}^{l}\left(\mathbf{h}_{E, i}^{l}\right)^{H} \widehat{\mathbf{h}}_{l k}^{l} \\
N_{t}^{N^{\infty}} \operatorname{tr}\left(\widehat{\mathbf{R}}_{l k}^{l} \mathbf{h}_{E, j}^{l}\left(\mathbf{h}_{E, i}^{l}\right)^{H}\right)=\left(\mathbf{h}_{E, i}^{l}\right)^{H} \widehat{\mathbf{R}}_{l k}^{l} \mathbf{h}_{E, j}^{l} \\
=\mathbf{e}_{i}^{H}\left(\mathbf{R}_{E, R}^{l}\right)^{1 / 2}\left(\mathbf{G}_{E}^{l}\right)^{H}\left(\mathbf{R}_{E, T}^{l}\right)^{1 / 2} \widehat{\mathbf{R}}_{l k}^{l} \\
\quad \times\left(\mathbf{R}_{E, T}^{l}\right)^{1 / 2} \mathbf{G}_{E}^{l}\left(\mathbf{R}_{E, R}^{l}\right)^{1 / 2} \mathbf{e}_{j} \\
\stackrel{N_{t} \rightarrow \infty}{\rightarrow}\left\{\mathbf{R}_{E, R}^{l}\right\}_{i j} \operatorname{tr}\left(\mathbf{R}_{E, T}^{l} \widehat{\mathbf{R}}_{l k}^{l}\right),
\end{gathered}
$$

where (95) is obtained based on [52, Corollary 1] and [53, Eq. (102)]. 
For $m=k$, similar to $(85)$, we have

$$
\begin{aligned}
& \left(\mathbf{h}_{E, i}^{l}\right)^{H} \widehat{\mathbf{h}}_{l m}^{l}\left(\widehat{\mathbf{h}}_{l m}^{l}\right)^{H} \mathbf{h}_{E, j}^{l} \\
& \stackrel{N_{t} \rightarrow \infty}{\rightarrow} \tau^{2} \sum_{t=0}^{L} P_{t m}\left(\mathbf{h}_{E, i}^{l}\right)^{H} \mathbf{C}_{l m}^{l} \mathbf{h}_{t m}^{l}\left(\mathbf{h}_{t m}^{l}\right)^{H}\left(\mathbf{C}_{l m}^{l}\right)^{H} \mathbf{h}_{E, j}^{l} \\
& +\tau^{2} \frac{P_{E}}{N_{e}} \sum_{r=1}^{N_{e}} \sum_{t=1}^{N_{e}}\left(\mathbf{h}_{E, i}^{l}\right)^{H} \mathbf{C}_{l m}^{l} \mathbf{h}_{E, r}^{l}\left(\mathbf{h}_{E, t}^{l}\right)^{H}\left(\mathbf{C}_{l m}^{l}\right)^{H} \mathbf{h}_{E, j}^{l} \\
& \quad+\left(\mathbf{h}_{E, i}^{l}\right)^{H} \mathbf{C}_{l m}^{l} \mathbf{\Omega}_{m} \mathbf{n n}^{H} \mathbf{\Omega}_{m}^{H}\left(\mathbf{C}_{l m}^{l}\right)^{H} \mathbf{h}_{E, j}^{l} .
\end{aligned}
$$

Then, based on [52, Corollary 1] and [53, Eq. (102)], we obtain

$$
\begin{gathered}
\left(\mathbf{h}_{E, i}^{l}\right)^{H} \mathbf{C}_{l m}^{l} \mathbf{h}_{E, r}^{l}\left(\mathbf{h}_{E, t}^{l}\right)^{H}\left(\mathbf{C}_{l m}^{l}\right)^{H} \mathbf{h}_{E, j}^{l} \\
=\mathbf{e}_{i}^{H}\left(\mathbf{R}_{E, R}^{l}\right)^{1 / 2}\left(\mathbf{G}_{E}^{l}\right)^{H}\left(\mathbf{R}_{E, T}^{l}\right)^{1 / 2} \\
\quad \times \mathbf{C}_{l m}^{l}\left(\mathbf{R}_{E, T}^{l}\right)^{1 / 2} \mathbf{G}_{E}^{l}\left(\mathbf{R}_{E, R}^{l}\right)^{1 / 2} \mathbf{e}_{r} \\
\quad \times \mathbf{e}_{t}^{H}\left(\mathbf{R}_{E, R}^{l}\right)^{1 / 2}\left(\mathbf{G}_{E}^{l}\right)^{H}\left(\mathbf{R}_{E, T}^{l}\right)^{1 / 2}\left(\mathbf{C}_{l m}^{l}\right)^{H} \\
\quad \times\left(\mathbf{R}_{E, R}^{l}\right)^{1 / 2} \mathbf{G}_{E}^{l}\left(\mathbf{R}_{E, T}^{l}\right)^{1 / 2} \mathbf{e}_{j} \\
\stackrel{N_{t} \rightarrow \infty}{\rightarrow}\left\{\mathbf{R}_{E, R}^{l}\right\}_{i r}\left\{\mathbf{R}_{E, R}^{l}\right\}_{t j} \operatorname{tr}\left(\mathbf{C}_{l m}^{l} \mathbf{R}_{E, T}^{l}\right) \operatorname{tr}\left(\left(\mathbf{C}_{l m}^{l}\right)^{H} \mathbf{R}_{E, T}^{l}\right) \\
=\left\{\mathbf{R}_{E, R}^{l}\right\}_{i r}\left\{\mathbf{R}_{E, R}^{l}\right\}_{t j}\left|\operatorname{tr}\left(\mathbf{C}_{l m}^{l} \mathbf{R}_{E, T}^{l}\right)\right|^{2} .
\end{gathered}
$$

Similarly, we have

$$
\begin{gathered}
\sum_{r=1}^{N_{e}} \sum_{t=1}^{N_{e}}\left(\mathbf{h}_{E, i}^{l}\right)^{H} \mathbf{C}_{l m}^{l} \mathbf{h}_{E, r}^{l}\left(\mathbf{h}_{E, t}^{l}\right)^{H}\left(\mathbf{C}_{l m}^{l}\right)^{H} \mathbf{h}_{E, j}^{l} \\
\stackrel{N_{t} \rightarrow \infty}{\rightarrow} \sum_{r=1}^{N_{e}}\left\{\mathbf{R}_{E, R}^{l}\right\}_{i r} \sum_{r=1}^{N_{e}}\left\{\mathbf{R}_{E, R}^{l}\right\}_{r j}\left|\operatorname{tr}\left(\mathbf{C}_{l m}^{l} \mathbf{R}_{E, T}^{l}\right)\right|^{2} \\
\left(\mathbf{h}_{E, i}^{l}\right)^{H} \mathbf{C}_{l m}^{l} \boldsymbol{\Omega}_{m} \mathbf{n} \mathbf{n}^{H} \mathbf{\Omega}_{m}^{H}\left(\mathbf{C}_{l m}^{l}\right)^{H} \mathbf{h}_{E, j}^{l} \\
\stackrel{N_{t} \rightarrow{ }^{\infty}}{\rightarrow} N_{0} \tau\left\{\mathbf{R}_{E, R}^{l}\right\}_{i j} \operatorname{tr}\left(\mathbf{R}_{E, T}^{l} \mathbf{C}_{l m}^{l}\left(\mathbf{C}_{l m}^{l}\right)^{H}\right) .
\end{gathered}
$$

Combining (23), (91)-(99), we have

$$
\mathbf{Q}^{N_{t} \rightarrow \infty} \mathbf{Q}_{\text {asy }}
$$

Based on (100), we obtain

$$
\begin{aligned}
\mathbf{w}_{0 m}^{H} \mathbf{H}_{E}^{0} \mathbf{Q}^{-1}\left(\mathbf{H}_{E}^{0}\right)^{H} \mathbf{w}_{0 m} & \stackrel{N_{t} \rightarrow \infty}{\rightarrow} \frac{1}{\left\|\widehat{\mathbf{h}}_{0 m}^{0}\right\|^{2}}\left(\widehat{\mathbf{h}}_{0 m}^{0}\right)^{H} \mathbf{H}_{E}^{0} \mathbf{Q}_{\text {asy }}^{-1}\left(\mathbf{H}_{E}^{0}\right)^{H} \widehat{\mathbf{h}}_{0 m}^{0},
\end{aligned}
$$

which can be further simplified using

$$
\frac{1}{N_{t}}\left\|\widehat{\mathbf{h}}_{0 m}^{0}\right\|^{2} \stackrel{N_{t} \rightarrow \infty}{\rightarrow} \frac{1}{N_{t}} \operatorname{tr}\left(\widehat{\mathbf{R}}_{0 m}^{0}\right)
$$

and

$$
\begin{aligned}
& \left(\widehat{\mathbf{h}}_{0 m}^{0}\right)^{H} \mathbf{H}_{E}^{0} \mathbf{Q}_{\text {asy }}^{-1}\left(\mathbf{H}_{E}^{0}\right)^{H} \widehat{\mathbf{h}}_{0 m}^{0} \\
& =\sum_{i=1}^{N_{e}} \sum_{j=1}^{N_{e}}\left\{\mathbf{Q}_{\text {asy }}^{-1}\right\}_{i j}\left(\widehat{\mathbf{h}}_{0 m}^{0}\right)^{H}\left(\mathbf{h}_{E, i}^{0}\right)\left(\mathbf{h}_{E, j}^{0}\right)^{H} \widehat{\mathbf{h}}_{0 m}^{0} \\
& =\sum_{i=1}^{N_{e}} \sum_{j=1}^{N_{e}}\left\{\mathbf{Q}_{\text {asy }}^{-1}\right\}_{i j}\left(\mathbf{h}_{E, i}^{0}\right)^{H} \widehat{\mathbf{h}}_{0 m}^{0}\left(\widehat{\mathbf{h}}_{0 m}^{0}\right)^{H} \mathbf{h}_{E, j}^{0} \\
& \stackrel{N_{t} \rightarrow \infty}{\rightarrow} \sum_{i=1}^{N_{e}} \sum_{j=1}^{N_{e}}\left\{\mathbf{Q}_{\text {asy }}^{-1}\right\}_{i j} \eta_{i j}^{0},
\end{aligned}
$$

which was obtained by following a similar approach as was used to obtain (100). Substituting (102) and (103) into (22) completes the proof.

APPENDIX C

\section{PROOF OF THEOREM 3}

When $p=\frac{1}{K}, \frac{1+\mathrm{SINR}_{0 m} \text {, asy }}{1+\mathrm{SINR}_{\text {eve, asy }}}$ can be simplified to

$$
\frac{1+\mathrm{SINR}_{0 m, \text { asy }}}{1+\mathrm{SINR}_{\text {eve, asy }}}=\frac{\gamma \theta_{b, p}+\gamma \theta_{m}+1}{\left(\gamma \theta_{b, p}+1\right)\left(\gamma \tilde{\theta}_{e}+1\right)} .
$$

The derivative of (104) with respect to $\gamma$ is given by

$$
\begin{aligned}
& \frac{d\left(\frac{\gamma \theta_{b, p}+\gamma \theta_{m}+1}{\left(\gamma \theta_{b, p}+1\right)\left(\gamma \tilde{\theta}_{e}+1\right)}\right)}{d \gamma} \\
& =\frac{-\left(\theta_{b, p}+\theta_{m}\right) \theta_{b, p} \tilde{\theta}_{e} \gamma^{2}-2 \gamma \theta_{b, p} \theta_{m}+\theta_{m}-\tilde{\theta}_{e}}{\left(\gamma \theta_{b, p}+1\right)^{2}\left(\gamma \tilde{\theta}_{e}+1\right)^{2}} .
\end{aligned}
$$

From (27) and (28), we observe that $\theta_{m} \geq 0$ and $\theta_{b, p} \geq 0$.

Next, we prove $\tilde{\theta}_{e} \geq 0$. From (33), we obtain

$$
\begin{aligned}
& \sum_{i=1}^{N_{e}} \eta_{i i}^{0} \\
& =\tau^{2} \sum_{i=1}^{N_{e}}\left\{\mathbf{R}_{E, R}^{0}\right\}_{i i} \sum_{t=0}^{L} P_{t m} \operatorname{tr}\left(\mathbf{R}_{E, T}^{0} \mathbf{C}_{0 m}^{0} \mathbf{R}_{t m}^{0}\left(\mathbf{C}_{0 m}^{0}\right)^{H}\right) \\
& +\tau^{2} \frac{P_{E}}{N_{e}}\left|\operatorname{tr}\left(\mathbf{C}_{0 m}^{0} \mathbf{R}_{E, T}^{0}\right)\right|^{2} \sum_{i=1}^{N_{e}} \sum_{r=1}^{N_{e}}\left\{\mathbf{R}_{E, R}^{0}\right\}_{i r} \sum_{r=1}^{N_{e}}\left\{\mathbf{R}_{E, R}^{0}\right\}_{r i} \\
& +N_{0} \tau \operatorname{tr}\left(\mathbf{R}_{E, T}^{0} \mathbf{C}_{0 m}^{0}\left(\mathbf{C}_{0 m}^{0}\right)^{H}\right) \sum_{i=1}^{N_{e}}\left\{\mathbf{R}_{E, R}^{0}\right\}_{i i}
\end{aligned}
$$

Since $\mathbf{R}_{E, R}^{0}$ is a receive correlation matrix, it is a Hermitian positive-semidefinite matrix. Therefore, we have $\left\{\mathbf{R}_{E, R}^{0}\right\}_{i i} \geq$ 0 . As a result, we obtain

$$
\tau^{2}\left\{\mathbf{R}_{E, R}^{0}\right\}_{i i} \sum_{t=0}^{L} P_{t m} \operatorname{tr}\left(\mathbf{R}_{E, T}^{0} \mathbf{C}_{0 m}^{0} \mathbf{R}_{t m}^{0}\left(\mathbf{C}_{0 m}^{0}\right)^{H}\right) \geq 0
$$

$$
N_{0} \tau\left\{\mathbf{R}_{E, R}^{0}\right\}_{i i} \operatorname{tr}\left(\mathbf{R}_{E, T}^{0} \mathbf{C}_{0 m}^{0}\left(\mathbf{C}_{0 m}^{0}\right)^{H}\right) \geq 0
$$


Also, we have

$$
\begin{aligned}
\sum_{i=1}^{N_{e}} \sum_{r=1}^{N_{e}}\left\{\mathbf{R}_{E, R}^{0}\right\}_{i r} \sum_{r=1}^{N_{e}}\left\{\mathbf{R}_{E, R}^{0}\right\}_{r i} & =\mathbf{1}_{N_{e}}^{H} \mathbf{R}_{E, R}^{0} \mathbf{R}_{E, R}^{0} \mathbf{1}_{N_{e}} \\
& =\left(\mathbf{R}_{E, R}^{0} \mathbf{1}_{N_{e}}\right)^{H} \mathbf{R}_{E, R}^{0} \mathbf{1}_{N_{e}} c \\
& \geq 0
\end{aligned}
$$

From (36), (106)-(109), we obtain $\tilde{\theta}_{e} \geq 0$. As a result, the equation

$$
-\left(\theta_{b, p}+\theta_{m}\right) \theta_{b, p} \tilde{\theta}_{e} \gamma^{2}-2 \gamma \theta_{b, p} \theta_{m}+\theta_{m}-\tilde{\theta}_{e}=0
$$

has at least one non-positive root. We assume that $\gamma_{1}$ and $\gamma_{2}, \gamma_{1}<\gamma_{2}$ are the two roots of (110) and $\gamma_{1} \leq 0$. Since $-\left(\theta_{b, p}+\theta_{m}\right) \theta_{b, p} \tilde{\theta}_{e}<0$, we have

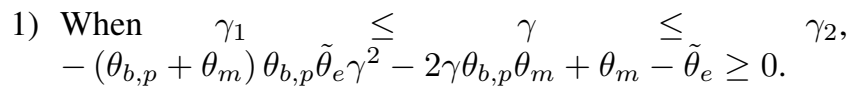

2) When $\gamma<\gamma_{1}$ or $\gamma \quad \gamma_{2}$, $-\left(\theta_{b, p}+\theta_{m}\right) \theta_{b, p} \tilde{\theta}_{e} \gamma^{2}-2 \gamma \theta_{b, p} \theta_{m}+\theta_{m}-\tilde{\theta}_{e}<0$.

Since $\gamma_{1} \leq 0, \gamma<\gamma_{1}$ can not hold. When $\gamma>\gamma_{2}$, we know from (105)

$$
\frac{d\left(\frac{\gamma \theta_{b, p}+\gamma \theta_{m}+1}{\left(\gamma \theta_{b, p}+1\right)\left(\gamma \tilde{\theta}_{e}+1\right)}\right)}{d \gamma}<0
$$

Finally, setting $\gamma_{\mathrm{th}}=\gamma_{2}$ in Theorem 3 completes the proof.

\section{APPENDIX D}

PROOF OF THEOREM 4

Since $\mathbf{R}_{t m}^{l}$ and $\mathbf{R}_{E, T}^{l}$ are positive semi-definite correlation matrices, $\sum_{t=0}^{L} \operatorname{tr}\left(\mathbf{R}_{t m}^{l} \mathbf{R}_{E, T}^{l}\right)=0$ is equivalent to $\sum_{t=0}^{L} \mathbf{R}_{t m}^{l} \mathbf{R}_{E, T}^{l}=\mathbf{0}$. For $\sum_{t=0}^{L} \mathbf{R}_{t m}^{l} \mathbf{R}_{E, T}^{l}=\mathbf{0}$, we have (112) at the top of the next page, where $\stackrel{(a)}{=}$ follows from the matrix inversion lemma [54]. Similarly, we can prove that when $\sum_{t=0}^{L} \mathbf{R}_{t m}^{l} \mathbf{R}_{E}^{l}=\mathbf{0}, \mathbf{C}_{l m}^{l}$ reduces to $\mathbf{C}_{l m \text {,orth }}^{l}$.

Also, when $\sum_{t=0}^{L} \mathbf{R}_{t m}^{l} \mathbf{R}_{E, T}^{l}=\mathbf{0}$, we have

$$
\mathbf{R}_{E, T}^{l}\left(\mathbf{C}_{l m}^{l}\right)^{H}=\mathbf{R}_{E, T}^{l} \mathbf{R}_{l m}^{l}\left(\mathbf{I}_{N_{t}}+\tau \sum_{t=0}^{L} P_{t k} \mathbf{R}_{t k}^{l}\right)^{-1}=\mathbf{0}
$$

and as a result,

$$
\begin{aligned}
& \operatorname{tr}\left(\mathbf{R}_{0 m}^{l} \mathbf{C}_{l m}^{l} \mathbf{R}_{E, T}^{l}\left(\mathbf{C}_{l m}^{l}\right)^{H}\right)=0, \\
& \operatorname{tr}\left(\mathbf{R}_{E, T}^{l} \mathbf{C}_{l m}^{l}\left(\mathbf{C}_{l m}^{l}\right)^{H}\right)=0, \\
& \operatorname{tr}\left(\mathbf{R}_{E, T}^{l} \mathbf{C}_{l m}^{l}\right)=0 .
\end{aligned}
$$

Substituting (114) into (33), yields

$$
\eta_{i j}^{l}=0
$$

The proof is completed by substituting (112)-(117) into (24).
APPENDIX E

PROOF OF THEOREM 5

When $N_{e}=1$, (26) reduces to

$$
\mathrm{SINR}_{\mathrm{eve}, \text { asy }}=\frac{p \gamma \theta_{e, e}}{q \gamma \theta_{e, q}+1} .
$$

Hence, maximizing $R_{\mathrm{sec}}$, asy in (24) is equivalent to maximizing

$$
\frac{1+\operatorname{SINR}_{0 m, \text { asy }}}{1+\mathrm{SINR}_{\text {eve, asy }}}=\frac{a_{1} p^{2}+b_{1} p+c_{1}}{a_{2} p^{2}+b_{2} p+c_{2}} .
$$

By taking the derivative of (119) with respect to $p$, we obtain

$$
\begin{aligned}
& \frac{d\left(\frac{a_{1} p^{2}+b_{1} p+c_{1}}{a_{2} p^{2}+b_{2} p+c_{2}}\right)}{d p} \\
& =\frac{\left(a_{1} b_{2}-a_{2} b_{1}\right) p^{2}+2\left(a_{1} c_{2}-a_{2} c_{1}\right) p+b_{1} c_{2}-b_{2} c_{1}}{\left(a_{2} p^{2}+b_{2} p+c_{2}\right)^{2}} .
\end{aligned}
$$

The proof is completed by setting (120) to zero and finding the solution.

\section{APPENDIX F \\ PROOF OF THEOREM 7}

Based on Theorems 2 and 5, the optimal asymptotic secrecy rate for the MF-AN design is given by

$$
R_{\text {sec, asy }}^{*}=\log _{2} \frac{a_{3} \gamma^{2}+b_{3} \gamma+1}{a_{4} \gamma^{2}+b_{4} \gamma+1} .
$$

Following a similar approach as in Appendix B, we obtain the asymptotic secrecy rate of the NS design as

$$
R_{\text {sec, asy }, \text { null }}=\log _{2} \frac{a_{5} \gamma+1}{a_{6} \gamma+1} .
$$

Performing some simplifications, $R_{\text {sec, asy }}^{*} \gtreqless R_{\text {sec, asy, null }}$ is equivalent to

$$
a_{7} \gamma^{3}+b_{7} \gamma^{2}+c_{7} \gamma \gtreqless 0 .
$$

Then, Theorem 7 is obtained by finding the feasible region of (123).

\section{APPENDIX G \\ PROOF OF THEOREM 8}

Following a similar approach as in Appendix B, when $L=$ $0, K=1, N_{e}=1$, and $N_{t} \rightarrow \infty$, SINR 01 , asy in (25) becomes (124), which is given at the top of the next page.

Also, SINR eve, asy $_{\text {in (26) becomes }}$

$\mathrm{SINR}_{\text {eve, asy }}=\frac{p \gamma \Lambda}{q \gamma\left(\operatorname{tr}\left(\mathbf{R}_{E, T}^{0}\right) \operatorname{tr}\left(\widehat{\mathbf{R}}_{01}^{0}\right)-\Lambda\right)+\operatorname{tr}\left(\widehat{\mathbf{R}}_{01}^{0}\right)}$.

For secure communication, we require SINR $_{01 \text {, asy }}>$

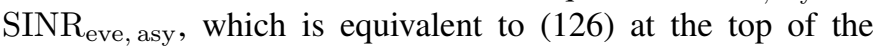
next page. 


$$
\begin{aligned}
\widehat{\mathbf{R}}_{l m}^{l} & =P_{l m} \tau \mathbf{R}_{l m}^{l}\left(N_{0} \mathbf{I}_{N_{t}}+\tau\left(\sum_{t=0}^{L} P_{t k} \mathbf{R}_{t k}^{l}+P_{E} r_{E, R}^{l} \mathbf{R}_{E, T}^{l}\right)\right)^{-1} \mathbf{R}_{l m}^{l} \\
& =P_{l m} \tau \mathbf{R}_{l m}^{l}\left(\left(\sqrt{N_{0}} \mathbf{I}_{N_{t}}+\frac{\tau}{\sqrt{N_{0}}} \sum_{t=0}^{L} P_{t k} \mathbf{R}_{t k}^{l}\right)\left(\sqrt{N_{0}} \mathbf{I}_{N_{t}}+\frac{\tau P_{E} r_{E, R}^{l}}{\sqrt{N_{0}}} \mathbf{R}_{E, T}^{l}\right)\right)^{-1} \mathbf{R}_{l m}^{l} \\
& =P_{l m} \tau \mathbf{R}_{l m}^{l}\left(\sqrt{N_{0}} \mathbf{I}_{N_{t}}+\frac{\tau P_{E} r_{E, R}^{l}}{\sqrt{N_{0}}} \mathbf{R}_{E, T}^{l}\right)^{-1}\left(\sqrt{N_{0}} \mathbf{I}_{N_{t}}+\frac{\tau}{\sqrt{N_{0}}} \sum_{t=0}^{L} P_{t k} \mathbf{R}_{t k}^{l}\right)^{-1} \mathbf{R}_{l m}^{l} \\
& \stackrel{(a)}{=} P_{l m} \tau \mathbf{R}_{l m}^{l}\left(\frac{1}{\sqrt{N_{0}}} \mathbf{I}_{N_{t}}-\frac{\tau P_{E} r_{E, R}^{l}}{\sqrt{N_{0}^{3}}} \mathbf{R}_{E, T}^{l}\left(\mathbf{I}_{N_{t}}+\frac{\tau P_{E} r_{E, R}^{l}}{N_{0}} \mathbf{R}_{E, T}^{l}\right)^{-1}\right)\left(\sqrt{N_{0}} \mathbf{I}_{N_{t}}+\frac{\tau}{\sqrt{N_{0}}} \sum_{t=0}^{L} P_{t k} \mathbf{R}_{t k}^{l}\right)^{-1} \mathbf{R}_{l m}^{l} \\
& =P_{l m} \tau \mathbf{R}_{l m}^{l}\left(\mathbf{I}_{N_{t}}+\tau \sum_{t=0}^{L} P_{t k} \mathbf{R}_{t k}^{l}\right)^{-1} \mathbf{R}_{l m}^{l}
\end{aligned}
$$

$$
\operatorname{SINR}_{01, \text { asy }}=\frac{p \gamma\left(\operatorname{tr}^{2}\left(\widehat{\mathbf{R}}_{01}^{0}\right)+\operatorname{tr}\left(\left(\mathbf{R}_{01}^{0}-\widehat{\mathbf{R}}_{01}^{0}\right) \widehat{\mathbf{R}}_{01}^{0}\right)\right)}{q \gamma \operatorname{tr}\left(\left(\mathbf{R}_{01}^{0}-\widehat{\mathbf{R}}_{01}^{0}\right) \widehat{\mathbf{R}}_{01}^{0}\right)+q \gamma \operatorname{tr}\left(\widehat{\mathbf{R}}_{01}^{0}\right) \operatorname{tr}\left(\mathbf{R}_{01}^{0}-\widehat{\mathbf{R}}_{01}^{0}\right)+\operatorname{tr}\left(\widehat{\mathbf{R}}_{01}^{0}\right)}
$$

$$
\frac{p \gamma\left(\operatorname{tr}^{2}\left(\widehat{\mathbf{R}}_{01}^{0}\right)+\operatorname{tr}\left(\left(\mathbf{R}_{01}^{0}-\widehat{\mathbf{R}}_{01}^{0}\right) \widehat{\mathbf{R}}_{01}^{0}\right)\right)}{q \gamma \operatorname{tr}\left(\left(\mathbf{R}_{01}^{0}-\widehat{\mathbf{R}}_{01}^{0}\right) \widehat{\mathbf{R}}_{01}^{0}\right)+q \gamma \operatorname{tr}\left(\widehat{\mathbf{R}}_{01}^{0}\right) \operatorname{tr}\left(\mathbf{R}_{01}^{0}-\widehat{\mathbf{R}}_{01}^{0}\right)+\operatorname{tr}\left(\widehat{\mathbf{R}}_{01}^{0}\right)}>\frac{p \gamma \Lambda}{q \gamma\left(\operatorname{tr}\left(\mathbf{R}_{E, T}^{0}\right) \operatorname{tr}\left(\widehat{\mathbf{R}}_{01}^{0}\right)-\Lambda\right)+\operatorname{tr}\left(\widehat{\mathbf{R}}_{01}^{0}\right)}
$$

When $N_{t} \rightarrow \infty$ and $N_{e}=1$, we have

$$
\begin{gathered}
\left\|\mathbf{h}_{E, 1}^{0}\right\|^{2}\left\|\widehat{\mathbf{h}}_{01}^{0}\right\|^{2} \stackrel{N_{t} \rightarrow \infty}{\rightarrow} \operatorname{tr}\left(\mathbf{R}_{E, T}^{0}\right) \operatorname{tr}\left(\widehat{\mathbf{R}}_{01}^{0}\right) \\
\left(\mathbf{h}_{E, 1}^{0}\right)^{H} \widehat{\mathbf{h}}_{01}^{0}\left(\widehat{\mathbf{h}}_{01}^{0}\right)^{H} \mathbf{h}_{E, 1}^{0} \stackrel{N_{t} \rightarrow \infty}{\rightarrow} \Lambda .
\end{gathered}
$$

Exploiting the Cauchy-Schwarz inequality, we obtain

$$
\left(\mathbf{h}_{E, 1}^{0}\right)^{H} \widehat{\mathbf{h}}_{01}^{0}\left(\widehat{\mathbf{h}}_{01}^{0}\right)^{H} \mathbf{h}_{E, 1}^{0} \leq\left\|\mathbf{h}_{E, 1}^{0}\right\|^{2}\left\|\widehat{\mathbf{h}}_{01}^{0}\right\|^{2} .
$$

Therefore, we have

$$
\operatorname{tr}\left(\mathbf{R}_{E, T}^{0}\right) \operatorname{tr}\left(\widehat{\mathbf{R}}_{01}^{0}\right)-\Lambda \geq 0
$$

Moreover, based on (5), we have

$$
\begin{aligned}
& \mathbf{R}_{01}^{0}-\widehat{\mathbf{R}}_{01}^{0} \\
& =\mathbf{R}_{01}^{0}\left(N_{0} \mathbf{I}_{N_{t}}+\tau\left(P_{01} \mathbf{R}_{01}^{0}+P_{E} \mathbf{R}_{E, T}^{0}\right)\right)^{-1} \\
& \quad \times\left(N_{0} \mathbf{I}_{N_{t}}+\tau P_{E} \mathbf{R}_{E, T}^{0}\right) \\
& =\mathbf{R}_{01}^{0}\left(\mathbf{I}_{N_{t}}+\tau P_{01} \mathbf{R}_{01}^{0}\left(N_{0} \mathbf{I}_{N_{t}}+\tau P_{E} \mathbf{R}_{E, T}^{0}\right)^{-1}\right)^{-1} .
\end{aligned}
$$

Since $\mathbf{R}_{01}^{0}$ and $\mathbf{R}_{E, T}^{0}$ are transmit correlation matrices, they are Hermitian positive-semidefinite matrices. From (5) and (131), we observe

$$
\operatorname{tr}\left(\widehat{\mathbf{R}}_{01}^{0}\right)>0, \quad \operatorname{tr}\left(\mathbf{R}_{01}^{0}-\widehat{\mathbf{R}}_{01}^{0}\right)>0
$$

Eqs. (130) and (132) indicate that the denominators of both the left hand and the right hand terms in (126) are positive.
Then, simplifying (126), we obtain (69). This completes the proof.

\section{APPENDIX H}

Proof of THEOREM 9 (10)

Setting $\mathbf{R}_{01}^{0}=\beta_{01} \mathbf{I}_{N_{t}}$ and $\mathbf{R}_{E, T}^{0}=\beta_{E} \mathbf{I}_{N_{t}}$, we have from

$$
\begin{aligned}
& \widehat{\mathbf{R}}_{01}^{0}=\frac{\tau P_{01} \beta_{01}^{2}}{N_{0}+\tau\left(P_{01} \beta_{01}+P_{E} \beta_{E}\right)} \mathbf{I}_{N_{t}} \\
& \operatorname{tr}\left(\widehat{\mathbf{R}}_{01}^{0}\right)=\frac{\tau P_{01} \beta_{01}^{2} N_{t}}{N_{0}+\tau\left(P_{01} \beta_{01}+P_{E} \beta_{E}\right)} \\
& \operatorname{tr}\left(\left(\mathbf{R}_{01}^{0}-\widehat{\mathbf{R}}_{01}^{0}\right) \widehat{\mathbf{R}}_{01}^{0}\right)=\frac{\tau P_{01} \beta_{01}^{3}\left(N_{0}+\tau P_{E} \beta_{E}\right) N_{t}}{\left(N_{0}+\tau\left(P_{01} \beta_{01}+P_{E} \beta_{E}\right)\right)^{2}} \\
& \operatorname{tr}\left(\mathbf{R}_{01}^{0}-\widehat{\mathbf{R}}_{01}^{0}\right)=\frac{\beta_{01}\left(N_{0}+\tau P_{E} \beta_{E}\right) N_{t}}{N_{0}+\tau\left(P_{01} \beta_{01}+P_{E} \beta_{E}\right)} \\
& \Lambda=\frac{\tau P_{01} \beta_{01}^{2} \beta_{E} N_{t}}{\left(N_{0}+\tau\left(P_{01} \beta_{01}+P_{E} \beta_{E}\right)\right)^{2}} \\
& \operatorname{tr}\left(\mathbf{R}_{E, T}^{0}\right)=\beta_{E} N_{t} .
\end{aligned}
$$

Substituting (133)-(138) into $\eta_{1}$ in (70), recalling $N_{t} \rightarrow \infty$, and simplifying, we obtain

$$
\eta_{1}=N_{t}^{4}\left(\tau P_{01} \beta_{01}+\tau P_{E} \beta_{E}+N_{0}\right)\left(P_{01} \beta_{01}-P_{E} \beta_{E}\right) .
$$

This completes the proof. 


\section{REFERENCES}

[1] T. L. Marzetta, "Noncooperative cellular wireless with unlimited numbers of base station antennas," IEEE Trans. Wireless Commun., vol. 9, pp. 3590-3600, Nov. 2010.

[2] J. Jose, A. Ashikhmin, T. L. Marzetta, and S. Vishwanath, "Pilot contamination and precoding in multi-cell TDD systems," IEEE Trans. Wireless Commun., vol. 10, pp. 2640-2651, Aug. 2011.

[3] H. Yin, D. Gesbert, M. Filippou, and Y. Liu, "A coordinated approach to channel estimation in large-scale multiple-antenna systems," IEEE J. Sel. Areas Commun., vol. 31, pp. 264-273, Feb. 2013.

[4] A. Adhikary, J. Nam, J.-Y. Ahn, and G. Caire, "Joint spatial division and multiplexing-The large-scale array regime," IEEE Trans. Inf. Theory, vol. 59, pp. 6441-6463, Oct. 2013.

[5] E. G. Larsson, O. Edfors, F. Tufvesson, and T. L. Marzetta, "Massive MIMO for next generation wireless systems," IEEE Commun. Mag. vol. 52, pp. 186-195, Feb. 2014

[6] H. Yin, D. Gesbert, and L. Cottatellucci, "Dealing with interference in distributed large-scale MIMO systems: A statistical approach," IEEE J. Sel. Topics Signal Process., vol. 8, pp. 942-953, Oct. 2014.

[7] J. Nam, A. Adhikary, J.-Y. Ahn, and G. Caire, "Joint spatial division and multiplexing: Opportunistic beamforming, user grouping and simplified downlink scheduling," IEEE J. Sel. Topics Signal Process., vol. 8, pp. 876-890, Oct. 2014.

[8] C. Sun, X. Gao, S. Jin, M. Matthaiou, Z. Ding, and C. Xiao, "Beam division multiple access transmission for massive MIMO communications," IEEE Trans. Commun., vol. 63, pp. 2170-2184, Jun. 2015.

[9] X. Meng, X. Gao, and X.-G. Xia, "Beam division multiple access transmission for massive MIMO communications," IEEE Trans. Commun., vol. 64, pp. 174-186, Jan. 2016.

[10] A. Adhikary, E. A. Safadi, M. Samimi, R. Wang, G. Caire, T. S. Rappaport, and A. F. Molisch, "Joint spatial division and multiplexing for mm-wave channels," IEEE J. Sel. Areas Commun., vol. 32, pp. 12391255, Jun. 2014.

[11] H. Q. Ngo, E. G. Larsson, and T. L. Marzetta, "Energy and spectral efficiency of very large multiuser MIMO systems," IEEE Trans. Commun., vol. 61, pp. 1436-1449, Apr. 2013.

[12] Y. Wu, C.-K. Wen, C. Xiao, X. Gao, and R. Schober, "Linear precoding for the MIMO multiple access channel with finite alphabet inputs and statistical CSI," IEEE Trans. Wireless. Commun., vol. 14, pp. 983-997, Feb. 2015.

[13] A. Lu, X. Gao, Y. Zheng, and C. Xiao, "Low complexity polynomial expansion detector with deterministic equivalents of the moments of channel Gram matrix for massive MIMO uplink," IEEE Trans. Commun., vol. 64, pp. 586-600, Feb. 2016.

[14] H. Huh, S.-H. Moon, Y.-T. Kim, I. Lee, and G. Caire, "Multi-cell MIMO downlink with cell cooperation and fair scheduling: A large-system limit analysis," IEEE Trans. Inf. Theory, vol. 57, pp. 7771-7786, Dec. 2011

[15] S. Jin, X. Wang, Z. Li, K.-K. Wong, Y. Huang, and X. Tang, "On massive MIMO zero-forcing transceiver using time-shifted pilots," IEEE Trans. Vehicular Technology, vol. 65, pp. 59-74, Jan. 2016.

[16] D. W. K. Ng, E. S. Lo, and R. Schober, "Energy-efficient resource allocation in OFDMA systems with large numbers of base station antennas," IEEE Trans. Wireless Commun., vol. 11, pp. 3292-3304, Sep. 2012.

[17] L. You, X. Gao, A. L. Swindlehurst, and W. Zhong, "Channel acquisition for massive MIMO-OFDM with adjustable phase shift pilots," IEEE Trans. Signal Process., vol. 64, pp. 1461-1476, Mar. 2016.

[18] S. Wu, L. Kuang, Z. Ni, J. Lu, D. Huang, and Q. Guo, "Low-complexity iterative detection for large-scale multiuser MIMO-OFDM systems using approximate message passing," IEEE J. Sel. Topics Signal Process., vol. 8, pp. 902-915, Oct. 2014.

[19] L. Dai, B. Wang, Y. Yuan, S. Han, C.-L. I, and Z. Wang, "Nonorthogonal multiple access for 5G: Solutions, challenges, opportunities, and future research trends," IEEE Commun. Mag., vol. 53, pp. 74-81, Sep. 2015.

[20] S. K. Mohammed and E. G. Larsson, "Per-antenna constant envelope precoding for large multi-user MIMO systems," IEEE Trans. Commun., vol. 61, pp. 1059-1071, Mar. 2013.

[21] B. Schnerier, "Cryptographic design vulnerabilities," IEEE Comput., vol. 9, pp. 585-595, Nov.-Dec. 1998.

[22] A. D. Wyner, "The wiretap channel," Bell Syst. Tech. J., vol. 54, pp. 1355-1387, Oct. 1975.

[23] I. Csiszár and J. Körner, "Broadcast channels with confidential messages," IEEE Trans. Inf. Theory, vol. 24, pp. 339-348, May 1978.
[24] A. Khisti and G. W. Wornell, "Secure transmission with multiple antennas I: The MISOME wiretap channel," IEEE Trans. Inf. Theory, vol. 56, pp. 3088-3104, Jul. 2010.

[25] — "Secure transmission with multiple antennas-Part II: The MIMOME wiretap channel," IEEE Trans. Inf. Theory, vol. 56, pp. 55155532, Nov. 2010.

[26] F. Oggier and B. Hassibi, "The secrecy capacity of the MIMO wiretap channel," IEEE Trans. Inf. Theory, vol. 57, pp. 4961-4972, Aug. 2011.

[27] Y. Wu, C. Xiao, Z. Ding, X. Gao, and S. Jin, "Linear precoding for finite alphabet signaling over MIMOME wiretap channels," IEEE Trans. Veh. Technol., vol. 61, pp. 2599-2612, Jul. 2012

[28] D. W. K. Ng, E. S. Lo, and R. Schober, "Robust beamforming for secure communication in systems with wireless information and power transfer," IEEE Trans. Wireless Commun., vol. 13, pp. 4599-4615, Aug. 2014.

[29] D. W. K. Ng and R. Schober, "Secure and green SWIPT in distributed antenna networks with limited backhaul capacity," IEEE Trans. Wireless Commun., vol. 14, pp. 5082-5097, Sep. 2015.

[30] X.Chen, C. Zhong, C. Yuen, and H.-H. Chen, "Multi-antenna relay aided wireless physical layer security," IEEE Communications Magazine, vol. 53, pp. 40-46, Dec. 2015

[31] F. Zhu, F. Gao, and M. Yao, "Zero-forcing beamforming for physical layer security of harvesting wireless communications," EURASIP J. Wireless Commun. and Networking, vol. 58, pp. 1-9, Dec. 2015.

[32] J. Zhang, C. Yuen, C.-K. Wen, S. Jin, K.-K. Wong, and H. Zhu, "Large system secrecy rate analysis for SWIPT MIMO wiretap channels," IEEE Trans. Inf. Forensics and Secur., vol. 11, pp. 74 - 85, Jan. 2016.

[33] S. Goel and R. Negi, "Guaranteeing secrecy using artificial noise," IEEE Trans. Wireless Commun., vol. 6, pp. 2180-2189, Jun. 2008.

[34] X. Zhou and M. R. McKay, "Secure transmission wiretap with artificial noise over fading channels: Achievable rate and optimal power allocation," IEEE Trans. Veh. Technol., vol. 59, pp. 3831-3842, Oct. 2010.

[35] J. Huang and A. L. Swindlehurst, "Robust secure transmission in MISO channels based on worst-case optimization," IEEE Trans. Signal Process., vol. 60, pp. 1696-1707, Apr. 2012.

[36] J. Wang, J. Lee, F. Wang, and T. Q. S. Quek, "Jamming-aided secure communication in massive MIMO Rician channels," IEEE Trans. Wireless Commun., vol. 14, pp. 6854-6868, Dec. 2015.

[37] H.-M. Wang, C. Wang, and D. W. K. Ng, "Artificial noise assisted secure transmission under training and feedback," IEEE Trans. Signal Process., vol. 63, pp. 6285-6298, Dec. 2015.

[38] W. Shen, L. Dai, B. Shim, S. Mumtaz, and Z. Wang, "Joint CSIT acquisition based on low-rank matrix completion for FDD massive MIMO systems," IEEE Commun. Lett., vol. 19, pp. 2178-2181, Dec. 2015.

[39] X. Chen, L. Lei, H. Zhang, and C. Yuen, "Large-scale MIMO relaying techniques for physical layer security: AF or DF?" IEEE Trans. Wireless Commun., vol. 14, pp. 5135-5146, Sep. 2015.

[40] J. Zhu, R. Schober, and V. K. Bhargava, "Secure transmission in multicell massive MIMO systems," IEEE Trans. Wireless Commun., vol. 13, pp. 4766-4781, Sep. 2014

[41] J. Zhu, R. Schober, and V. K. Bhargava, "Linear precoding of data and artificial noise in secure massive MIMO systems," IEEE Trans. Wireless Commun., vol. 15, pp. 2245-2261, Mar. 2016.

[42] H.-M. Wang, T.-X. Zheng, J. Yuan, D. Towsley, and M. H. Lee, "Physical layer security in heterogeneous cellular networks," IEEE Trans. Commun., vol. 64, pp. 1204-1219, Mar. 2016

[43] X. Zhou, B. Maham, and A. Hjørungnes, "Pilot contamination for active eavesdropping," IEEE Trans. Wireless Commun., vol. 11, pp. 903-907, Mar. 2012.

[44] D. Kapetanović, G. Zheng, K.-K. Wong, and B. Ottersten, "Detection of pilot contamination attack using random training and massive MIMO," in Proc. IEEE Personal, Indoor, and Mobile Radio Communications (PIMRC 13), London, U.K., Sep. 2013, pp. 13-18.

[45] S. Im, H. Jeon, J. Choi, and J. Ha, "Secret key agreement under an active attack in MU-TDD systems with large antenna arrays," in Proc. IEEE Global. Telecommun. Conf. (GLOBECOM 2013), Atlanta, USA, Dec. 2013, pp. 1849-1855.

[46] Y. O. Basciftci, C. E. Koksal, and A. Ashikhmin, "Securing massive MIMO at the physical layer," [Online]. Available: http://arxiv.org/abs/1505.00396.

[47] Y. Wu, S. Jin, X. Gao, M. R. McKay, and C. Xiao, "Transmit designs for the MIMO broadcast channel with statistical CSI," IEEE Trans. Signal Process., vol. 62, pp. 4451-4446, Sep. 2014.

[48] X. Gao, L. Dai, S. Han, C.-L. I, and R. W. Heath, "Energy-efficient hybrid analog and digital precoding for mmWave MIMO systems with large antenna arrays," to appear in IEEE J Sel. Areas Commun.. 
[49] H. Shin and J. H. Lee, "Capacity of multiple-antenna fading channels: Spatial fading correlation, double scattering, and keyhole," IEEE Trans. Inf. Theory, vol. 49, pp. 2636-2647, Oct. 2003.

[50] T. Kailath, A. H. Sayed, and B. Hassibi, Linear Estimation. New Jersey: Prentice Hall, 2000.

[51] Y. S. Cho, J. Kim, W. Y. Yang, and C. G. Kang, MIMO-OFDM Wireless Communications with MATLAB. Singapore: John Wiley \& Sons (Asia) Pte Ltd, 2010.

[52] J. Evans and D. N. C. Tse, "Large system performance of linear multiuser receivers in multipath fading channels," IEEE Trans. Inf. Theory, vol. 46, pp. 2059-2078, Sep. 2000.

[53] C.-K. Wen, G. Pan, K.-K. Wong, M. Guo, and J.-C. Chen, "A deterministic equivalent for the analysis of non-Gaussian correlated MIMO multiple access channels," IEEE Trans. Inf. Theory, vol. 59, pp. 329352, Jan. 2013.

[54] D. Bernstein, Matrix Mathematics. Princeton: Princeton University Press, 2005. 